\title{
Measurements of Wide-Band Cochlear Reflectance in Humans
}

\author{
Daniel M. Rasetshwane ${ }^{1}$ and Stephen T. Neely ${ }^{1}$ \\ ${ }^{1}$ Boys Town National Research Hospital, 555 North 30th Street, Omaha, NE 68131, USA
}

Received: 16 January 2012; Accepted: 21 May 2012; Online publication: 12 June 2012

\begin{abstract}
The total sound pressure measured in the ear canal may be decomposed into a forward- and a reversepropagating component. Most of the reverse-propagating component is due to reflection at the eardrum. However, a measurable contribution to the reversepropagating component comes from the cochlea. Otoacoustic emissions (OAEs) are associated with this component and have been shown to be important noninvasive probes of cochlear function. Total earcanal reflectance (ECR) is the transfer function between forward and reverse propagating components measured in the ear canal. Cochlear reflectance (CR) is the inner-ear contribution to the total ECR, which is the measured OAE normalized by the stimulus. Methods are described for measuring CR with a wide-band noise stimulus. These measurements offer wider bandwidth and minimize the influence of the measurement system while still maintaining features of other OAEs (i.e., frequency- and level-dependent latency). CR magnitude decreases as stimulus level increases. Envelopes of individual band-limited components of the time-domain CR have multiple peaks with latencies that persist across stimulus level, despite a shift in group delay. CR has the potential to infer cochlear function and status, similar to other OAE measurements.
\end{abstract}

Keywords: otoacoustic emissions, latency, impedance, gammatone

Correspondence to: Daniel M. Rasetshwane - Boys Town National Research Hospital 555 North 30th Street, Omaha, NE 68131, USA. Telephone: +1-402-498-6372; fax: +1-402-498-6351; email: Daniel. Rasetshwane@boystown.org

\section{INTRODUCTION}

Sound pressure measured in the ear canal may be regarded as being composed of two components: (1) a forward-propagating component that transports acoustic energy into the inner ear for the purpose of hearing and (2) a reverse-propagating component that is produced in response to the forward component. In human ears, much of the reverse-propagating component comes from the eardrum. However, a small but measurable contribution to the reversepropagating component comes from the cochlea, the primary sensory organ for hearing, which is located within the inner ear. We refer to the transfer function between forward- and reverse-propagating components measured in the ear canal as ear-canal reflectance (ECR). We refer to the inner-ear contribution to this reflectance, which is equivalent to a measurement of otoacoustic emission (OAE) normalized by the stimulus, as cochlear reflectance (CR). In this study, methods to extract CR from measurements of ECR are described. The CR extraction method includes two steps: (1) subtraction of high-level ECR (with mostly linear cochlear response) from low-level ECR (with both linear and nonlinear responses) and (2) timefrequency analysis. The objective is to obtain a cochlear response that has the least possible influence of the middle-ear and measurement system and that can be interpreted in terms of a linear model. CR measurements may provide alternative means for clinical prediction of supra-threshold cochlear status and for future cochlear modeling interpretations. Abbreviations and symbols used in this article are listed in Table 1.

Otoacoustic emissions are acoustical signals that originate within the cochlea as by-products of its normal signal-processing function and constitute the reversepropagating component of sound pressure that originates from the cochlea. OAEs may be generated either 
TABLE 1

List of abbreviations and symbols

\begin{tabular}{|c|c|}
\hline $\begin{array}{l}\text { Abbreviation or } \\
\text { symbol }\end{array}$ & Meaning \\
\hline ECR & Ear-canal reflectance \\
\hline CR & Cochlear reflectance \\
\hline WBN & Wideband noise \\
\hline LSC & Linear-swept frequency chirp \\
\hline TF & Transfer function \\
\hline$L$ & Stimulus level \\
\hline$r_{\mathrm{WBN}, L}(t)$ & $\begin{array}{l}\text { Time-domain reflectance measured using } \\
\text { wideband noise stimulus at stimulus level } L\end{array}$ \\
\hline$r_{\mathrm{LSC}}(t)$ & $\begin{array}{l}\text { Time-domain reflectance measured using } \\
\text { linear-swept frequency chirp }\end{array}$ \\
\hline$r_{\mathrm{CR}, L}(t)$ & $\begin{array}{l}\text { Time-domain cochlear reflectance } \\
\text { measured at stimulus level } L\end{array}$ \\
\hline$f_{\mathrm{C}}$ & Center frequency of gammatone filter \\
\hline $\operatorname{ERB}\left(f_{\mathrm{c}}\right)$ & $\begin{array}{l}\text { Equivalent rectangular bandwidth of } \\
\text { gammatone filter with center frequency } f_{\mathrm{C}}\end{array}$ \\
\hline$r_{\mathrm{CR}, L}\left(f_{\mathrm{C}}, t\right)$ & $\begin{array}{l}\text { Time-domain cochlear reflectance } \\
\text { measured at stimulus level } L \text { for gammatone } \\
\text { filter with center frequency } f_{\mathrm{c}}\end{array}$ \\
\hline$N_{L}$ & $\begin{array}{l}\text { Lower bound of cochlear reflectance in } \\
\text { time-frequency domain }\end{array}$ \\
\hline$N_{H}$ & $\begin{array}{l}\text { Upper bound of cochlear reflectance in } \\
\text { time-frequency domain }\end{array}$ \\
\hline$\tau_{\mathrm{CR}}\left(f_{\mathrm{C}}\right)$ & $\begin{array}{l}\text { Group delay in milliseconds of gammatone } \\
\text { filter with center frequency } f_{C}\end{array}$ \\
\hline$N_{\mathrm{CR}}\left(f_{\mathrm{C}}\right)$ & $\begin{array}{l}\text { Group delay in cycles of the center } \\
\text { frequency } f_{\mathrm{c}} \text { of gammatone filter with center } \\
\text { frequency } f_{\mathrm{c}}\end{array}$ \\
\hline$f_{a \mid b}$ & Break point frequency in group delay \\
\hline CRM & Cochlear reflectance magnitude \\
\hline $\mathrm{CRM}_{L}\left(f_{\mathrm{c}}\right)$ & $\begin{array}{l}\text { Cochlear reflectance magnitude as a } \\
\text { function of center frequency } f_{\mathrm{C}} \text { at stimulus } \\
\text { level } L\end{array}$ \\
\hline $\mathrm{CRM}_{L}$ & $\begin{array}{l}\text { Cochlear reflectance magnitude at stimulus } \\
\text { level } L \text {, obtained from } \mathrm{CRM}_{L}\left(f_{\mathrm{C}}\right) \text { by } \\
\text { summing across frequency }\end{array}$ \\
\hline
\end{tabular}

spontaneously, in the absence of any acoustical stimulation, or as a response evoked by stimulation. OAEs are generated by vibrations within the cochlea at various locations. These vibrations travel towards the base of the cochlea, through the middle ear, and to the ear canal where they can be detected as sound pressure. OAEs can be evoked using (1) a transient stimulus, (2) a pure tone, or (3) a pair of tones. The type of stimulus determines the OAE name. Stimuli for transient-evoked OAEs (TEOAE) include clicks (click-evoked OAE or CEOAE) and short-duration tone bursts and evoke a wider frequency range of OAE simultaneously (with clicks evoking a wider frequency range compared to tone bursts). Stimulus-frequency OAEs (SFOAEs) are evoked using pure tones and cover a narrow frequency range around the frequency of the stimulus. Distortionproduct OAEs (DPOAEs) are evoked using a pair of primary tones. OAEs have also been evoked using noise with a band-limited spectral density that is similar to that of a click used for evoking CEOAE (Maat et al. 2000).
OAEs can also be classified based on the mechanism or "source" of emission (e.g., Shera and Guinan 1999). It is generally accepted that there are two distinct OAE generation mechanisms-linear coherent reflection and nonlinear distortion (e.g., Shera and Guinan 1999). OAEs produced by these two mechanisms have different properties. For example, the phase of the linear coherent reflection component changes rapidly with frequency, while the phase of the nonlinear distortion component is essentially flat as a function of frequency (e.g., Shera and Guinan 1999; Kemp 2002; Dhar et al. 2011). Although it is generally agreed that linear coherent reflection is the primary mechanism involved in SFOAEs and that nonlinear distortion is the primary mechanism involved in DPOAEs, details of these OAE generation mechanisms are still a matter of debate (e.g., Siegel et al. 2005; Yates and Withnell 1999). Knowledge of the precise mechanism is important because it affects the particular processing and decoding of OAE data used to extract information relevant to the hearing process (Kemp 2002). The generation mechanisms may also correlate with particular cochlear pathologies (Shera and Guinan 1999), although empirical evidence in support of this view remains elusive.

CR is the cochlear contribution to the total earcanal reflectance measured in the ear canal. It is essentially the measured OAE normalized by the stimulus. Specifically, the OAE waveform is deconvolved by the forward pressure. Our definition of CR should not be confused with Shera et al. (2005) who define CR looking into the cochlea from the stapes. A complete characterization of reflectance, as with any transfer function, requires measurements across a wide frequency range. In linear, time-invariant systems, transfer functions are independent of the stimulus used to measure them. However, CR is stimulus dependent because cochlear nonlinearities are functionally significant. This study measured CR using a wide-band noise (WBN) stimulus over a range of stimulus levels. Use of a WBN stimulus allows for a characterization of the measurements as a transfer function and also offers other benefits as described later. These measurements have the potential to infer cochlea function and status, similar to OAE measurements.

Ear-canal reflectance (ECR) can be measured by placing a microphone and an appropriately calibrated sound source in the ear canal (e.g., Allen 1986; Keefe et al. 1992; Siegel 1994; Neely and Gorga 1998). Most of the reflected energy that constitutes ECR is linearly related to stimulus intensity and comes from the eardrum and middle ear (for a review, see Keefe and Schairer 2011); however, a small contribution, with both linear and nonlinear components, is due to reflection from the cochlea (Allen 1997). Allen described a method for 
measuring the nonlinear component of the ECR. They measured ECR at several different stimulus levels, using tonal stimuli and a calibrated sound source. By substituting a high-level impedance for the characteristic impedance when calculating reflectance, they extracted a nonlinear component of reflectance. Their results demonstrated that cochlear reflectance varied with stimulus level. However, the interpretation of tonal responses as a transfer function presents a theoretical problem because the interpretation of reflectance as a transfer function assumes a linear, time-invariant system. When the system is nonlinear, then it may no longer be valid to combine independent measurements made one frequency at a time and interpret the set of frequencies as if they represent a single linear system. Use of a WBN stimulus in the current measurements allows for the invocation of de Boer's (1997) nonlinear equivalence (EQ-NL) theorem, which in turn theoretically allows for the interpretation of the data using concepts and principles applicable to linear systems such as transfer functions, Fourier analysis, and timefrequency analysis. According to the EQ-NL theorem, for a given class of nonlinear system, of which the cochlea is an example, there is an equivalent linear system that has the same response as the nonlinear system for a WBN stimulus at a specific level.

This study describes methods for extracting CR from ECR. Our methods for extraction assume that noncochlear contributions to ECR vary linearly with stimulus level, while CR varies mainly nonlinearly with level. We demonstrate the level dependence of CR measurements and show that CR latency has frequency and level dependence that is a characteristic of signals of cochlea origin. Qualitative comparisons are made to previous measurement of transfer functions (TFs) of CEOAE and SFOAE (Kalluri and Shera 2007; Sisto and Moleti 2008). We apply time-frequency analysis to our estimate of time-domain CR to obtain frequency band-limited estimates that may be useful in probing the function and status of spatially limited regions of the cochlea. We examine envelopes of these band-limited CR envelopes and demonstrate that they have multiple peaks with delays that persist across stimulus level, despite the shift in group delays with level. This analysis may potentially provide more information regarding the generation sites of OAEs.

\section{METHODS}

\section{Subjects}

A total of 20 subjects with ages ranging from 15 to 65 years participated in this study. Subjects were required to have audiometric thresholds of $20 \mathrm{~dB}$ HL or better (ANSI 1996) for the octave and interoctave frequencies from 0.25 to $8 \mathrm{kHz}$. Middleear status was assessed using tympanometry with a 226$\mathrm{Hz}$ probe tone. To qualify for inclusion, the following tympanometric criteria had to be met: peak-compensated static admittance of $0.3-2.5 \mathrm{mmhos}$ and peak pressure between -100 and $+50 \mathrm{daPa}$. Otoscopic examination was also performed as a way to further ensure normality of the ear-canal and ear drum. All subjects were recruited from a database of potential research subjects that is maintained at Boys Town National Research Hospital. Subjects were paid for their participation. The study described in this article was conducted under an approved Institutional Review Board protocol. After first obtaining informed consent, then audiometric, tympanometric, and otoscopic assessments, data collection was initiated, which required an average of 72 min per subject.

\section{Measurements}

The sound-delivery system consisted of two modified tweeters (TW010F1, Audax, France) acoustically attached by plastic tubes to an ER-10B+ probe microphone (Etymotic Research, Elk Grove Village, IL, USA). Each stimulus condition was repeated on each sound source, and the independent measurements from the two sources were combined into a single average. Amplifiers placed between the soundcard and the tweeters provided power gain and reduced the electrical load on the soundcard output. (The design of the modified tweeters and amplifiers was developed at Northwestern University by J. H. Siegel, who generously shared a prototype with us.) The measurement system was calibrated prior to data collection to determine the Thévenin-equivalent source impedance and pressure (Allen 1986; Keefe et al. 1992; Rasetshwane and Neely 2011).

A WBN signal and a wide-band linear-swept frequency chirp (LSC) signal, both generated digitally at a sampling rate of $48 \mathrm{kHz}$, were used as stimuli. The duration of each stimulus/response buffer was $171 \mathrm{~ms}$. The WBN stimulus was presented at levels of 20-70 dB SPL in 10-dB steps, and the LSC stimulus was presented at $60 \mathrm{~dB}$ SPL. These stimulus levels were determined using a sound level meter (System 824, Larson Davis, Provo, UT, USA) with C weighting, and their range is similar to those routinely used for CEOAE (e.g., Kalluri and Shera 2007) and SFOAE (Choi et al. 2008) measures. The WBN stimulus had constant spectral amplitude and random phase; that is, it was white noise. White noise was chosen for compatibility with the EQ-NL theorem. These stimulus conditions are summarized in Table 2. The fourth column of the table reports the averaging time in seconds. More averaging was done at the lower stimulus levels to mitigate the effect of low signal/ 
TABLE 2

Stimulus types and levels

\begin{tabular}{llcc}
\hline Test & Stimulus & Level $(d B$ SPL) & Averaging time (s) \\
\hline 1 & WBN & 20 & 192 \\
2 & WBN & 30 & 96 \\
3 & WBN & 40 & 48 \\
4 & WBN & 50 & 24 \\
5 & WBN & 60 & 12 \\
6 & WBN & 70 & 6 \\
7 & LSC & 60 & 6 \\
\hline
\end{tabular}

Data were collected using wide-band noise (WBN) at levels of 20-70 dB SPL and using a wide-band linear-swept frequency chirp (LSC) at $60 \mathrm{~dB}$ SPL. More averaging was done at the lower stimulus levels to mitigate the effect of low SNR. Each test condition was repeated four times for additional SNR boosting. Averaging times are for a single test condition

noise ratio (SNR) due to the reduced magnitude of the response. Each test condition in Table 2 was repeated four times to further boost the SNR, as described later. The data collected with the LSC stimulus (test 7 in Table 2) are used as a reference signal for estimating CR as described later. Measurements of the response acoustic pressure for the different stimulus conditions were used to determine the ear-canal acoustic impedance from which the reflectance is determined. Stimulus delivery and data collection were controlled using locally developed software (EMAV; Neely and Liu 1994).

Reflectance calculations require accurate estimates of the characteristic impedance of the ear canal. If the crosssectional area $A$ of an ear canal is known, then the characteristic impedance $Z_{0}$ can be calculated using $Z_{0}=\rho c / A$, where $\rho$ is the density of air and $c$ is the speed of sound. However, it is not possible to measure the cross-sectional area of the ear canal accurately when the OAE probe is inserted in the ear canal. Using the crosssectional area of the calibration tube is only appropriate if it matches that of the ear canal. Assuming incorrect cross-sectional area, and hence incorrect characteristic impedance, can lead to errors in the calculation of reflectance. Our characteristic impedance estimation procedure avoids the need to know the cross-sectional area of the ear canal by determining the characteristic impedance from the load impedance. The procedure uses an iterative procedure that estimates the "surge" component of the load impedance by minimizing the time-domain ECR and its first derivative at $t=0$. This method is an improvement to our previous procedure (Rasetshwane and Neely 2011) that only minimized the time-domain ECR at $t=0$. Surge impedance characterizes the immediate response of an acoustic load to an impulsive stimulus and has been shown to accurately estimate the characteristic impedance of uniform tubes with various diameters (Scheperle et al. 2011). The improved surge estimation procedure provides better estimates of the characteristic impedance of uniform tubes when compared to the previous procedure.

\section{Estimation of cochlear reflectance}

Measurements of ear-canal impedance $Z_{\mathrm{ec}}$ and estimates of characteristic impedance $Z_{0}$ were used to calculate ECR in the frequency-domain using the formula

$$
R_{\mathrm{ec}}(x, s)=\frac{Z_{\mathrm{ec}}(x, s)-Z_{0}(x)}{Z_{\mathrm{ec}}(x, s)+Z_{0}(x)}
$$

where $s=i 2 \pi f$ is the Laplace complex frequency variable (e.g., Claerbout 1985). Frequency-domain ECRs were transformed to time-domain ECRs using the inverse Fourier transform. The frequency-domain reflectance was multiplied by a Blackman window with an $18-\mathrm{kHz}$ half-width prior to computation of the time-domain ECR (1) to reduce contribution from higher frequencies, where the measurement was less reliable, and (2) to eliminate ringing in the time domain. A Blackman window has less sideband leakage than equivalent-length Hamming and Hanning windows, which, in this case, reduces spread in the time-domain ECR. The validity of applying a Blackman window to the ECR was recently demonstrated by Rasetshwane and Neely (2011) by showing that the corresponding time-domain representation retains sufficient information to estimate ear-canal shape.

Estimation of CR is done in two stages. In the first stage, we take advantage of the dependence of cochlear reflectance on stimulus level to remove activity due to the middle ear by applying a subtraction procedure. Time-frequency analysis is used in the second stage to remove residual middle-ear activity that was not removed by the subtraction procedure and any measurement-system artifact. Let $r_{\mathrm{WBN}, L}(t)$ be the time-domain ECR elicited using WBN (tests 1-6 in Table 2) at stimulus level $L$ and let $r_{\text {LSC }}(t)$ be the time-domain ECR elicited using LSC (test 7 in Table 2). Most of the reflected energy in ECRs $r_{\mathrm{WBN}, L}(t)$ and $r_{\mathrm{LSC}}(t)$ is linearly related to stimulus intensity and comes from the middle ear; however, a small component is due to reflection from the cochlea. We obtain an estimate of the nonlinear time-domain $\mathrm{CR} r_{\mathrm{CR}, L}(t)$ at stimulus level $L$ as

$$
r_{C R, L}(t)=r_{\mathrm{WBN}, L}(t)-r_{\mathrm{LSC}}(t)
$$

This subtraction procedure is intended to remove time-domain reflectance contributions that vary linearly with level, especially contributions coming from the middle ear. The LSC stimulus provides an intense excitation in the cochlea that saturates most of the nonlinearities. The WBN stimulus at the highest stimulus intensity also provides intense excitation; however, the LSC stimulus, although changing in time, is more 
localized at any given instant and was found to produce better estimates of cochlear reflectance in preliminary analysis. The LSC response is thought to have less cochlear contribution than a WBN response at the same level because activity within the cochlea is more localized at any instant in time, due to the instantaneous frequency content of the stimulus, causing local vibration amplitudes to be larger and, therefore, causing contributions from nonlinear elements, which saturate at higher amplitudes, to be relatively smaller. Separate time-domain CRs were computed from the four measurements and averaged to improve the SNR. Additional SNR improvements were obtained by averaging the two time-domain CRs from the two sound sources. An estimate of the noise level was obtained as the difference between repeated measurements.

Figure 1 shows examples of the time-domain reflectances after the subtraction procedure for one subject at stimulus levels of 20-70 dB SPL of WBN stimulus. The ordinate label shows the stimulus level and not the level of the time-domain CR. High frequency content temporally precedes low-frequency content, consistent with signals of cochlea origin. The activity below $t=1 \mathrm{~ms}$ is residual middle-ear activity that was not removed by the subtraction procedure and measurement-system artifact. This activity is dominant below $t=1$ because the ear-canal timedomain reflectance has large amplitude for $t<1$ (see Rasetshwane and Neely 2011, Fig. 5).

To eliminate the residual middle-ear activity and measurement-system artifact time-frequency analysis of
$\mathrm{CR} r_{\mathrm{CR}, L}(t)$ was performed using a complex gammatone filterbank with 49 channels (e.g., Patterson and Holdsworth 1996; Hohmann 2002). The individual gammatone filters of the filterbank were of order $n=4$ and were implemented using the infinite impulse response algorithm of Härmä (1999). The filterbank was designed such that all the filters had the same tuning of $Q_{\mathrm{ERB}}=3$, with $Q_{\text {ERB }}$ defined as $f_{\mathrm{c}} / \mathrm{ERB}\left(f_{\mathrm{c}}\right)$, where $f_{\mathrm{c}}$ is the center frequency and $\operatorname{ERB}\left(f_{\mathrm{c}}\right)$ is the equivalent rectangular bandwidth of the filter with center frequency $f_{c}$, respectively (see Shera et al. 2010). The center frequencies were logarithmically spaced from $f_{\mathrm{c}}=0.0625-16 \mathrm{kHz}$ in 1/6-octave steps. The outputs of the gammatone filterbank $r_{\mathrm{CR}, L}\left(f_{\mathrm{c}}, t\right)$ are complex-valued bandpassfiltered time-domain components of the CR $r_{\mathrm{CR}, L}(t)$. The real part of the output of the filterbank represents the band-limited gammatone filter output, whereas the imaginary part approximates its Hilbert transform (Hohmann 2002). The use of complex-valued gammatone filters facilitates accurate calculation of the timedomain envelope since two signals, the imaginary and real parts, are involved in the computation of a single envelope as opposed to use of only one signal when the filter outputs are real. The individual outputs of the gammatone filterbank $r_{\mathrm{CR}},{ }_{L}\left(f_{\mathrm{c}}, t\right)$ can experience different delays, which can affect the estimation of the latencies of these outputs. Compensation for delay of the gammatone filters was performed by delaying the fine structure and the envelope of each filter's impulse response so that all channels have their envelope maximum and their fine-

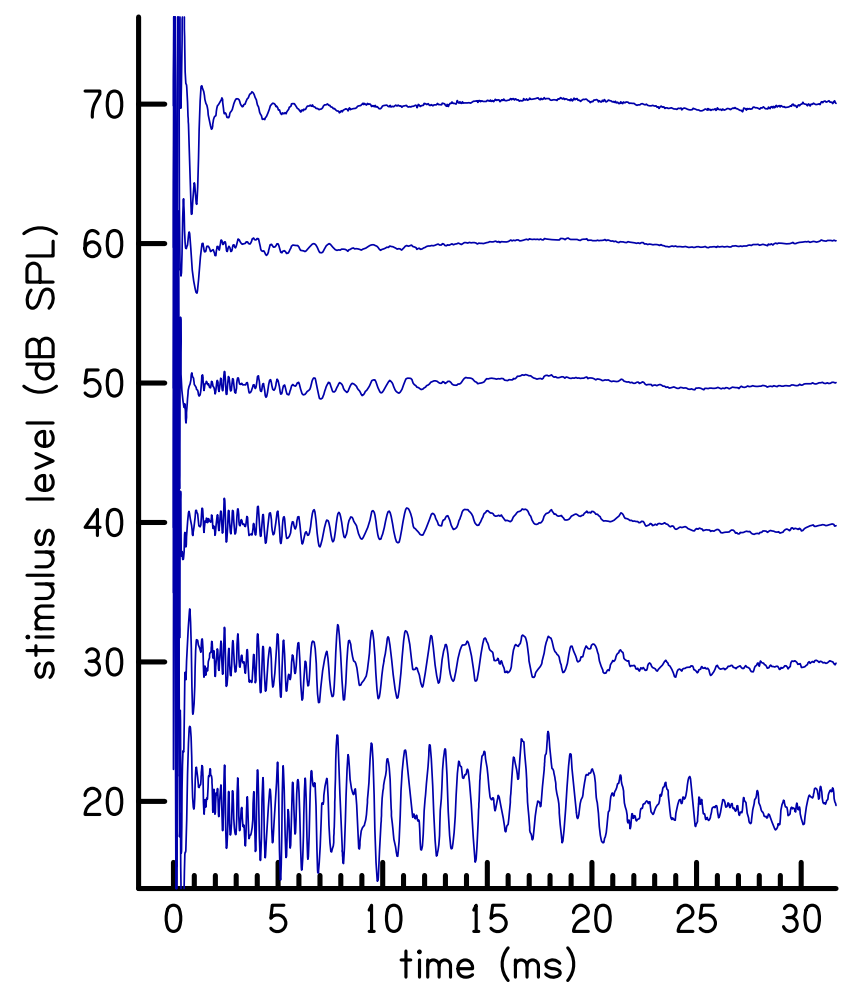

FIG. 1. Time-domain cochlear reflectance after the subtraction procedure and before the time-frequency analysis. The ordinate label shows the stimulus level and not the level of the time-domain CR. High-frequency content temporally precedes low-frequency content in the timedomain CR. The activity before $t=1 \mathrm{~ms}$ is middle-ear activity that was not removed by the subtraction procedure. 
structure maximum at the same time instant, the desired filterbank group delay (Hohmann 2002). The advantage of using the gammatone filterbank over a short-time Fourier transform or a continuous-wavelet transform is that it allows frequency resolution to be specified as desired at both low and high frequencies. Additionally, gammatone filters are often used in psychophysical auditory models (e.g., Patterson and Holdsworth 1996; Meddis et al. 2001; Jepsen et al. 2008) because of their similarity to physiological measures of basilar membrane vibrations (e.g., Rhode and Robles 1974).

An example of the time-frequency analysis of CR is illustrated in Figure 2 for one of the subjects at a stimulus level of $20 \mathrm{~dB}$ SPL, using a spectrogram. We refer to this spectrogram as the gammatone spectrogram. In the figure, more reddish color corresponds to larger magnitude compared to more bluish color and the magnitude range (from red to blue) is $108 \mathrm{~dB}$. Also plotted in Figure 2 are functions indicating constant numbers of delay cycles $\left(N \equiv t f_{\mathrm{c}}\right)$ :

$$
\begin{gathered}
N_{L} \equiv t f_{\mathrm{c}}=4+0.27 f \\
N_{H} \equiv t f_{\mathrm{c}}=40 .
\end{gathered}
$$

The $0.27-\mathrm{ms}$ factor in the definition of $N_{L}$ [cf. Eq. (3)] is the average round-trip transmission delay to the middle ear ossicles, determined from timedomain ECR (see Rasetshwane and Neely 2011,

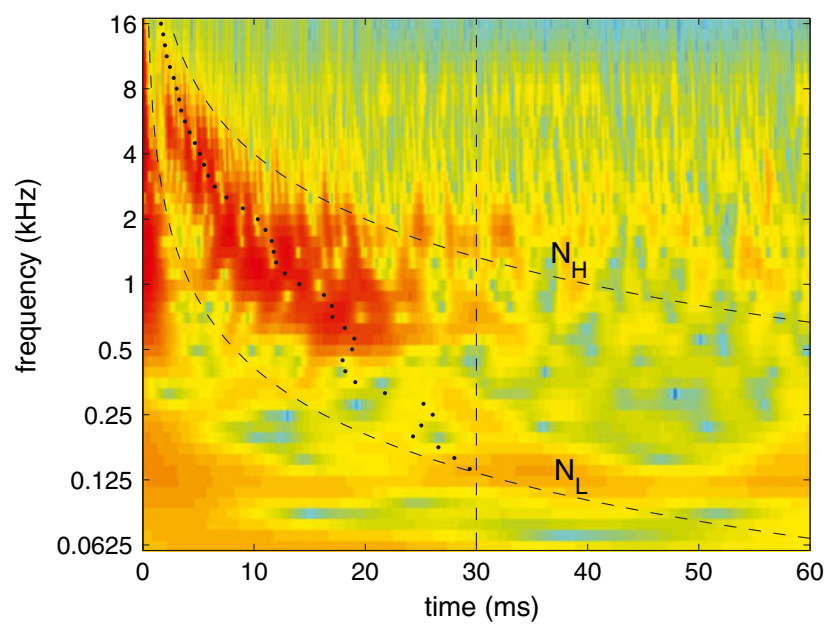

FIG. 2. Time-frequency analysis of CR using the gammatone spectrogram. The region of high energy of the gammatone spectrogram enclosed by functions $N_{L}$ and $N_{H}$ includes most of the exponential decaying energy of CR. The region below $N_{L}$ includes residual middle ear and measurement system activity that was not removed by the subtraction procedure. The activity beyond $t=30$ (indicated with vertical dashed line) is due to re-reflection of the traveling wave. The dots are estimates of the group delay.
Fig. 5). The region of high energy of the gammatone spectrogram enclosed by $N_{L}$ and $N_{H}$ includes most of the energy of the CR. The region below $N_{L}$ presumably includes residual middle-ear reflectance and measurement-system artifact that was not removed by the subtraction procedure of Eq. (2). However, at high frequencies (above $8 \mathrm{kHz}$ ), the separation of this activity from possible cochlear contributions from the base is not that clear. The activity beyond $t=30 \mathrm{~ms}$ or above $N_{H}$ is thought to be due to re-reflection of the traveling wave at the stapes, sound source, and the basilar membrane emission source. We estimate CR in the region enclosed by $N_{L}, N_{H}$, and $t=30 \mathrm{~ms}$. An inspection of all the data collected revealed that the limits $N_{L}, N_{H}$, and $t=30 \mathrm{~ms}$ were always a good bound of the exponential decaying cochlear contribution, and the limit $N_{L}$ was always a reasonable boundary between cochlear reflectance and residual middle-ear activity.

Figure 2 also provides information about CR latency. For example, the delay of the center of energy of any given frequency is visualized as the horizontal center of the reddish region along the corresponding vertical location. To estimate the CR latency for a given stimulus level $L$, we compute, for center frequency $f_{\mathrm{c}}$, the group delay $\tau_{\mathrm{CR}}\left(f_{\mathrm{c}}\right)$ of the components $r_{\mathrm{CR}, L}\left(f_{\mathrm{c}}, t\right)$

$$
\tau_{\mathrm{CR}}\left(f_{\mathrm{c}}\right)=\frac{\sum_{t} t\left|r_{\mathrm{CR}, L}\left(f_{\mathrm{c}}, t\right)\right|^{2}}{\sum_{t}\left|r_{\mathrm{CR}, L}\left(f_{\mathrm{c}}, t\right)\right|^{2}} .
$$

This definition of group delay, which is consistent with Goldstein et al. (1971), corresponds to the time at which the energy of $r_{\mathrm{CR}, L}\left(f_{\mathrm{c}}, t\right)$ is centered within the spectrogram region described above. The dots in Figure 2 are an example of the group-delay estimates $\tau_{\mathrm{CR}}\left(f_{\mathrm{c}}\right)$. The time limits in the summations are defined by the functions $N_{L}$ and $N_{H}$ :

$$
N_{L}<N<N_{H}
$$

and

$$
0 \leq t<30 \mathrm{~ms}
$$

The time limit $t=30 \mathrm{~ms}$ is larger than the largest previously reported latency measurement for signals of cochlea origin (see, e.g., Neely et al. 1988) and does not result in an underestimation of $\tau_{\mathrm{CR}}\left(f_{\mathrm{c}}\right)$. The time limits of Eqs. (6) and (7) can be interpreted as a windowing method similar to that commonly used for extracting OAEs (see, e.g., Kalluri and Shera 2007), with the added feature that the onset of the window 
(controlled by $N_{L}$ ) varies with frequency. An early onset time is used for high frequencies, allowing us to extract high frequency CR that has short delay, and a late onset time is used for low frequencies to reduce contribution from residual middle-ear reflectance and any measurement-system artifact.

Following Shera and Guinan (2003), we define

$$
N_{\mathrm{CR}}\left(f_{\mathrm{c}}\right) \equiv f_{\mathrm{c}} \tau_{\mathrm{CR}}\left(f_{\mathrm{c}}\right),
$$

a dimensionless group delay by measuring time in cycles or periods of the center frequency of the filters, $f_{\mathrm{c}}$. A power law fit, i.e., straight-line approximation on the $\log -\log$ axes, of the form

$$
y=a x^{b}
$$

can be fit to the group delay data $\tau_{\mathrm{CR}}\left(f_{\mathrm{c}}\right)$ or the function $N_{\mathrm{CR}}\left(f_{\mathrm{c}}\right)$ to characterize the frequency-latency relationship (e.g., Neely et al. 1988). In this equation, parameter $a$ is the latency at $f_{\mathrm{c}}=1 \mathrm{kHz}$ and $x=f_{\mathrm{c}} / 1 \mathrm{kHz}$.

The estimate of CR magnitude (CRM) as a function of frequency is obtained as the root mean square of the outputs of the gammatone filters $r_{\mathrm{CR}, L}\left(f_{\mathrm{c}}, t\right)$

$$
\mathrm{CRM}_{L}\left(f_{\mathrm{c}}\right)=\gamma \sqrt{\frac{1}{N} \sum_{t}\left|r_{\mathrm{CR}, L}\left(f_{\mathrm{c}}, t\right)\right|^{2}}
$$

where the time limits in the summation are again specified by Eqs. (6) and (7). The scale factor $\gamma$ given by

$$
\gamma=\sqrt{\frac{2 f_{\mathrm{s}}}{\operatorname{ERB}\left(f_{\mathrm{c}}\right)}},
$$

where $f_{\mathrm{s}}$ is the sampling rate and ERB $\left(f_{\mathrm{c}}\right)$ is as defined above, was applied to remove high frequency emphasis (introduced using bandwidths that were proportional to the center frequencies) and to provide for physical interpretation.

To demonstrate validity of our method for computing the reflectance magnitude [cf. Eq. (10)], Figure 3 compares the average ECR magnitude for the subjects included in this study computed from $r_{\text {LSC }}(t)$ using Eq. (10) to average ECR computed using the magnitude Fourier transform of $r_{\mathrm{LSC}}(t)$. There is agreement between the two methods; the reflectance magnitude obtained with Eq. (10) is a smooth version of the reflectance magnitude obtained as the magnitude Fourier transform. The smoothed spectrum is convenient when comparing spectra across different stimulus levels. The trends in the ECR magnitude of
Figure 3 are in agreement with recent ECR measurements reported by Rosowski et al. (2012), which are likewise in agreement with several previous measurements of ECR (e.g., Voss and Allen 1994).

An estimate of CRM as a function of stimulus level is obtained from Eq. (10) by integrating over frequency, i.e.

$$
\mathrm{CRM}_{L}=\frac{1}{N} \sum_{f_{c}} \operatorname{CRM}_{L}\left(f_{\mathrm{c}}\right)
$$

where the frequency limits in the summation are again specified by Eqs. (6) and (7).

\section{RESULTS}

Figure 4 shows the mean levels and \pm 1 standard deviation (SD) of time-domain $\mathrm{CR} r_{\mathrm{CR}, L}(t)$ after the subtraction procedure, as function of stimulus level for the subjects who participated in this study. The mean noise level, defined as the difference between repeated measurements of time-domain CR, is also shown. The levels of $r_{\mathrm{CR}, L}(t)$ are presented as an indicator of the SNR of our data and not as an indicator of the level of the OAE that would be obtained if $r_{\mathrm{CR}, L}(t)$ was convolved by the stimulus, since $r_{\mathrm{CR}, L}(t)$ include residual contributions from the middle-ear and measurement system. An indicator of the level of the OAE can be obtained from a reflectance that excludes middle-ear activity, as discussed later. The $r_{\mathrm{CR}, L}(t) \mathrm{SNR}$ is about $10 \mathrm{~dB}$ at stimulus levels of $20-50 \mathrm{~dB}$ and about $12 \mathrm{~dB}$ at higher stimulus levels. The positive SNR demonstrates the reliability of our measurements for all stimulus levels. The SDs for $r_{\mathrm{CR}, L}(t)$ describe intersubject variability. Some of this variability is expected from coherentreflection theory, due to the role of random mechanical irregularity in the process of otoacoustic emission generation (Shera et al. 2008), and some of the variability may be due to measurement noise.

\section{Cochlear reflectance magnitude}

Figure 5 shows examples of gammatone spectrograms of CR at six stimulus levels for the same subject whose CR at $20 \mathrm{~dB}$ SPL was shown in Figure 2. As stimulus level increases, the energy of the component that we associate with CR (between $N_{L}$ and $N_{H}$ ) decreases and the energy of the component in the region below $N_{L}$ remains the same or increases; that is why we associate the latter with the middle-ear and the measurement system. The temporal persistence of CR also depends on level; there is more re-reflected energy closer to $30 \mathrm{~ms}$ at the lower levels compared to the higher levels. 


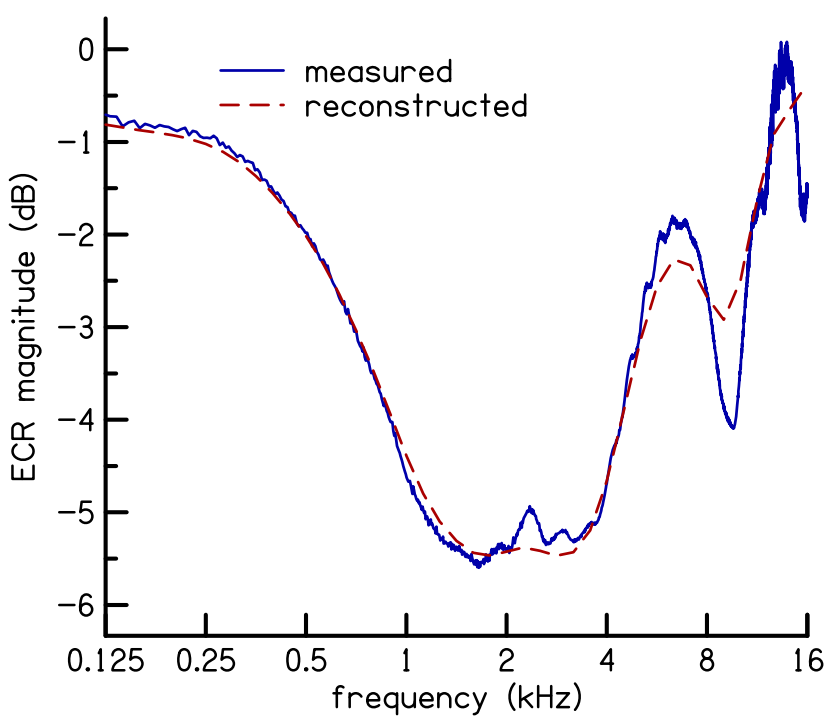

FIG. 3. Comparison of average ECR magnitude measured using Fourier transform (solid line) to ECR reconstructed from the gammatone-filter outputs (dashed line). The reconstructed ECR magnitude is a smooth version of the measured ECR magnitude. The agreement validates the gammatone-filterbank method for computing the CR magnitude [cf. Eq. (10)].

Cochlear reflectance magnitude (CRM) as a function of frequency, $\mathrm{CRM}_{L}\left(f_{\mathrm{c}}\right)$ [cf. Eq. (10)] for two subjects is plotted in Figure 6, for stimulus levels of 20-70 dB SPL. The dependence of CRM on stimulus level is visible-CRM is higher at lower stimulus levels. Individual CRM has spectral oscillations similar to

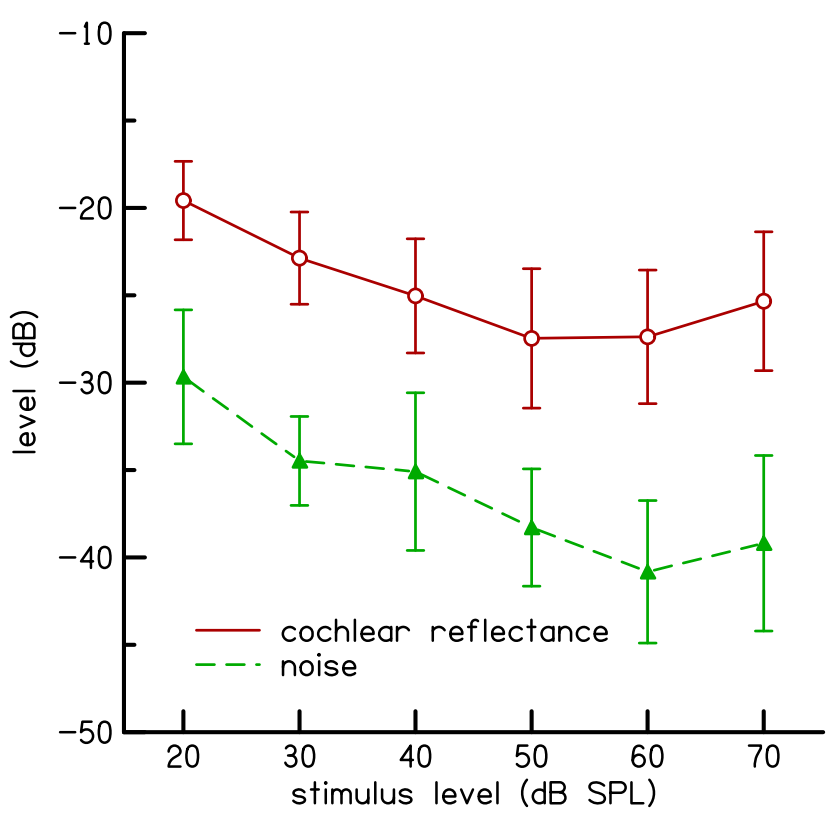

FIG. 4. Mean levels $\pm 1 \mathrm{SD}$ of $\mathrm{CR} r_{\mathrm{CR}, L}(t)$ (open circles) obtained with the subtraction procedure (before time-frequency analysis) and noise floor (filled triangles) as functions of stimulus level. The SNR (difference between the two curves) is near constant for stimulus levels of 20-50 dB and increases for higher stimulus levels. The positive SNR demonstrates the reliability of our measurements for all stimulus levels. those observed in CEOAE and SFOAE TFs by Kalluri and Shera (2007) and in CEOAE TFs by Sisto and Moleti (2008). However, the notches in the CRM spectra are not as sharp as those in the OAE TFs. Individual variation, in terms of location of peaks and notches, between the two subjects can also be observed.

Mean CRM for the entire group of subjects is plotted Figure 7. As expected, averaging over subjects removes the individual spectral oscillations but reveals global spectral features. Mean CRM has a bandpass characteristic with a broad peak centered at $1.1 \mathrm{kHz}$ at stimulus levels of 20-50 dB SPL. The spectral shape of $\mathrm{CRM}_{L}\left(f_{\mathrm{c}}\right)$ becomes flatter at 60 and $70 \mathrm{~dB}$ SPL. but the peak at $1.1 \mathrm{kHz}$ remains prominent. This peak, as well as the general spectral shape of our mean CRM, is similar to TEOAE TF of Sisto and Moleti (2008). Kalluri and Shera (2007) also observed this peak in their CEOAE and SFOAE transfer functions.

The overall dependence of CRM on level can also be revealed by integrating CRM functions of Figure 7 across frequency to obtain CRM as a function of stimulus level, $\mathrm{CRM}_{L}$ [cf. Eq. (12)]. Figure 8 shows $\mathrm{CRM}_{L}$ functions for individual subjects and the group mean $\mathrm{CRM}_{L}$. Although there is variability in individual subjects, the $\mathrm{CRM}_{L}$ functions all share the same qualitative form. They all decrease with increases in stimulus level, and most show saturation or onset of saturation at the highest stimulus level, i.e., further increase in stimulus level does not result in considerable decrease in CRM. At low level (from $L=20-30 \mathrm{~dB}$ SPL) mean $\mathrm{CRM}_{L}$ has a slope of $-0.48 \mathrm{~dB} / \mathrm{dB}$. This slope steepens to $-0.60 \mathrm{~dB} / \mathrm{dB}$ at intermediate levels (from $L=30-60 \mathrm{~dB}$ SPL). At high stimulus levels (from $L=60-70 \mathrm{~dB}$ SPL), mean $\mathrm{CRM}_{L}$ becomes independent of level and has a slope near zero $(-0.06 \mathrm{~dB} / \mathrm{dB})$. The slope of $\mathrm{CRM}_{L}$ at intermediate levels is similar to the range of slopes, -0.55 to -0.7 , of equivalent CEOAE TF analysis of Sisto and Moleti. Kalluri and Shera (2007) obtained a shallower slope of about -0.4 between $L=30$ and $60 \mathrm{~dB}$ SPL in their unified analysis of CEOAE and SFOAE TFs of a single subject, but interestingly as we did, they observed a declining slope (less steep) at low levels. However, their slope at low levels was much shallower than the slope we observed. The reduction in the slope of $\mathrm{CRM}_{L}$ at low level suggests onset of cochlear linearity.

The level of the OAE at each stimulus level can be deduced by deriving an OAE input/output (I/O) function from the $\mathrm{CRM}_{L}$ function and it ranges from $-5 \mathrm{~dB}$ SPL (at $L=20 \mathrm{~dB}$ SPL) to $26 \mathrm{~dB}$ SPL (at $L=$ $70 \mathrm{~dB}$ SPL). This OAE I/O function (plot not shown) has a slope of $0.40 \mathrm{~dB} / \mathrm{dB}$ between $L=30$ and $60 \mathrm{~dB}$ SPL and a slope of $0.52 \mathrm{~dB} / \mathrm{dB}$ between $L=20$ and $30 \mathrm{~dB}$ SPL. The OAE I/O slope of 0.40 is shallower than the mean slopes $(0.61$ and 0.65$)$ reported by 

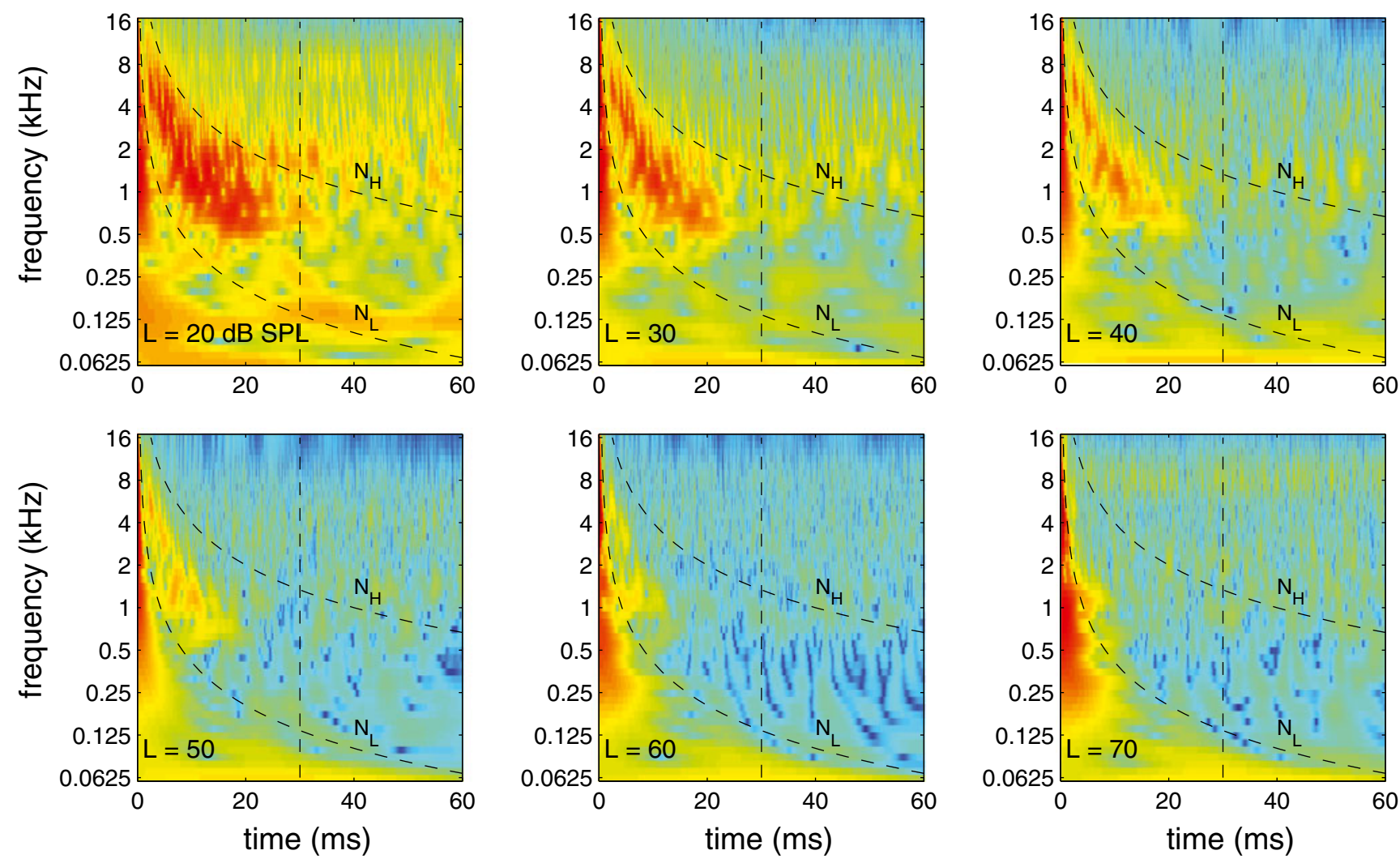

FIG. 5. Illustration of CR dependence on stimulus level $(L)$ and frequency using gammatone spectrograms. CR can be observed at the four lowest stimulus levels, and its magnitude decreases with increasing stimulus level. There is no evidence of CR at the highest level. CR persists longer at lower stimulus levels than higher levels; there is more re-reflected energy near $30 \mathrm{~ms}$ at the lowest level.

Choi et al. (2008) in their two different analyses of SFOAE I/O functions.

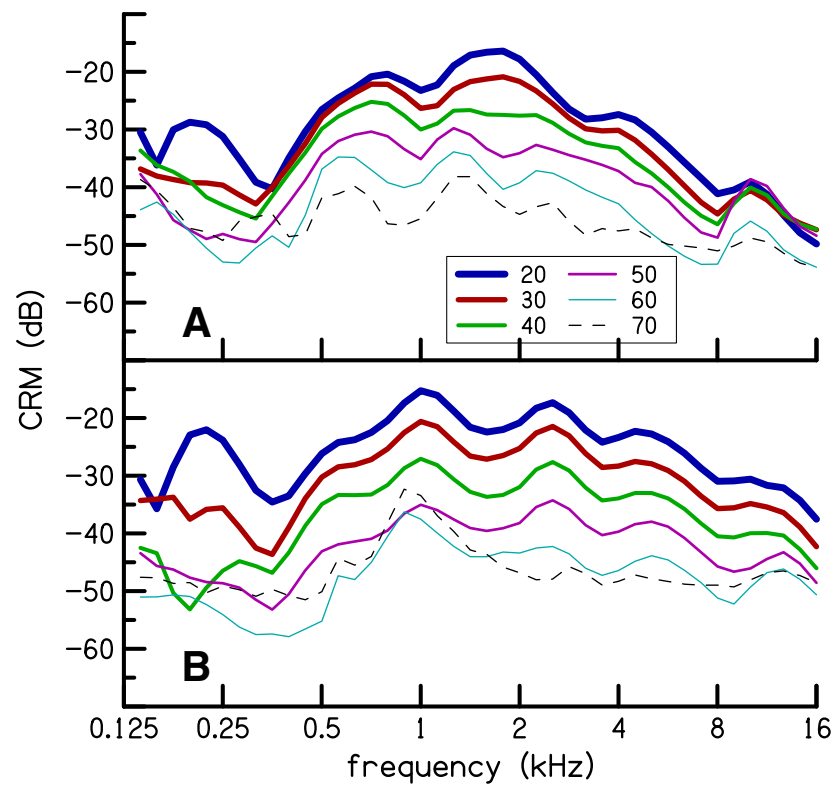

FIG. 6. Individual CR magnitude (CRM) as a function of frequency, $\mathrm{CRM}_{L}\left(f_{\mathrm{c}}\right)$ for two subjects, for stimulus levels of 20-70 dB SPL. CRM has spectral oscillations similar to those observed in CEOAE and SFOAE transfer functions by Kalluri and Shera (2007) and in CEOAE transfer functions by Sisto and Moleti (2008). Individual variation between the two subjects can also be observed.

\section{Reflectance latency}

\section{Latencies of time-domain components}

An example of the time-domain CR envelope is shown in Figure 9 for one subject for center frequency $f_{\mathrm{c}}=$ $4 \mathrm{kHz}$ and stimulus levels of 20-70 dB SPL. This analysis shows the variation in the envelope of the time-domain CR as a function of stimulus level at a given center frequency. In terms of the function $r_{\mathrm{CR}, \mathrm{L}}$ $\left(f_{\mathrm{c}}, t\right)$, Figure 9 shows $\left.r_{\mathrm{CR}, L}\left(f_{\mathrm{c}}=4, t\right)\right|_{L=20,30, \ldots 70 \mathrm{~dB}}$. The CR envelopes displayed in the right panel are normalized to the maximum peak for visual clarity, and the envelopes displayed in the left panel are not normalized to show the dependence of their amplitude on stimulus level. The dashed vertical lines are the limits $N_{L}$ and $N_{H}$ [cf. Eqs. (3) and (4)]. Superimposed on the plots are the delay of the dominant peak or envelope delay (filled squares) and group delay, $\tau_{\mathrm{CR}}\left(f_{\mathrm{c}}=4\right)$ (filled circles). It is interesting to note that the CR envelopes have multiple peaks at each level, not just the dominant peak and the timings of these peaks persist across level. The amplitudes of the peaks are large at low stimulus levels and decrease with increases in stimulus levels. Recall that a similar trend (decrease in CR with increase in stimulus level) was observed for the functions $\mathrm{CRM}_{L}\left(f_{c}\right)$ and $\mathrm{CRM}_{L}$. The peak at $t=1.3 \mathrm{~ms}$ is the dominant peak at $L=60$ 


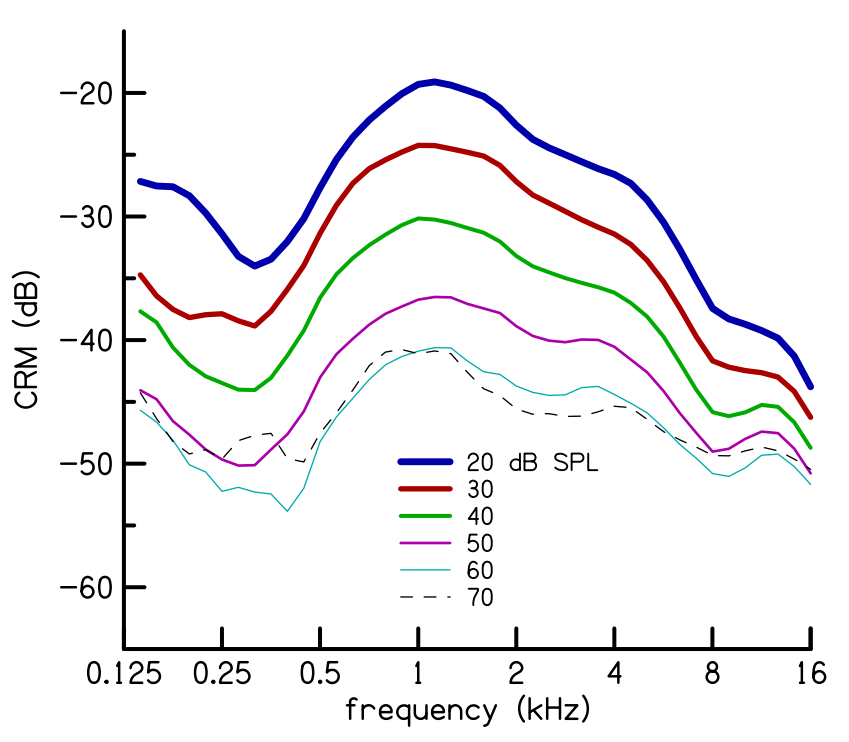

FIG. 7. Mean CR magnitude (CRM) as a function of frequency, $\mathrm{CRM}_{L}\left(f_{C}\right)$, for stimulus levels of 20-70 dB SPL. CRM has a bandpass characteristic with energy concentrated between 1 and $2 \mathrm{kHz}$, at the lower stimulus levels. CRM is larger at lower stimulus levels and saturates at high stimulus levels.

and $70 \mathrm{~dB}$ SPL. The peak at $t=4.2 \mathrm{~ms}$, which is small at $L=60$ and $70 \mathrm{~dB}$ SPL, becomes the dominant peak from $L=50$ to $30 \mathrm{~dB}$ SPL. That is, the dominant peak, as well as the group delay, shifts to later times as the stimulus level decreases.

The latencies and non-normalized amplitudes of the CR envelopes of Figure 9 are depicted in Figure 10 as functions of stimulus level. Only peaks with delays of up to the maximum of the envelope delay plus $2 \mathrm{~ms}$ are shown, to reduce clutter in the plot. Several features are noteworthy. The peaks at a given delay

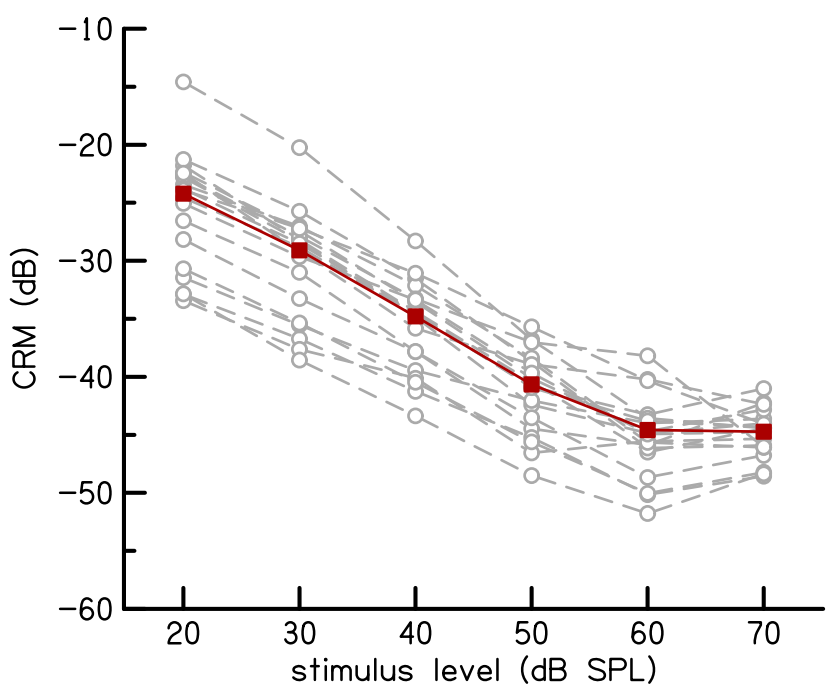

FIG. 8. CR magnitude as a function of stimulus level, $C R M_{L}$. $C R M_{L}$ for individual subjects (dashed lines with open circles) and mean for the subjects (solid line with filled squares) are shown. Mean $C \mathrm{M}_{L}$ has slopes of $-0.48 \mathrm{~dB} / \mathrm{dB}$ at low levels (from $L=20$ to $30 \mathrm{~dB} \mathrm{SPL}$ ), $-0.60 \mathrm{~dB} / \mathrm{dB}$ at intermediate levels (from $L=30$ to $60 \mathrm{~dB} \mathrm{SPL}$ ) and $-0.06 \mathrm{~dB} / \mathrm{dB}$ at high levels (from $L=60$ to $70 \mathrm{~dB} \mathrm{SPL}$ ). persist across stimulus level (see left panel), with only minor shifts in their timings at the higher stimulus levels. Superimposed on this panel are the envelope delay (filled squares) and group delay, $\tau_{\mathrm{CR}}\left(f_{\mathrm{c}}=4\right)$ (filled circles). At $L=20 \mathrm{~dB}$ SPL, the peak with a delay of $5.3 \mathrm{~ms}$ (hour-glass symbol) is the dominant peak. From $L=30$ to $50 \mathrm{~dB}$ SPL, the peak with a delay of $4.2 \mathrm{~ms}$ (diamond symbol) is the dominant peak, and at $L=60$ and $70 \mathrm{~dB}$ SPL, the peak with a delay of $1.3 \mathrm{~ms}$ (square symbol) is now the dominant peak. The group delay, like the envelope delay, decreases with increasing stimulus level, but assumes different values as explained later.

Another interesting observation is that the amplitude of the envelope peaks decrease with increases in stimulus level and that the dynamic range of the amplitude of earlier peaks is less than the dynamic range of the amplitude of later peaks. For example, the amplitude of the peak with a delay of $1.3 \mathrm{~ms}$ (square symbol) is $-10.3 \mathrm{~dB}$ (largest value) at $L=70 \mathrm{~dB}$ and $-22.6 \mathrm{~dB}$ (smallest value) at $L=60 \mathrm{~dB}$, a dynamic range of $12.3 \mathrm{~dB}$. In comparison, peaks with delays of $6.8 \mathrm{~ms}$ (picnic-table symbol) and $7.3 \mathrm{~ms}$ (upwardarrow symbol) have amplitudes with dynamic ranges of 33.3 and $35.8 \mathrm{~dB}$, respectively. In addition to having a wider dynamic range, the amplitudes of the later occurring delay peaks also have a lower threshold compared to the earlier occurring delay peaks; that is, low level stimulation produces large amplitude. The shift in envelope delay with increasing stimulus levels can also be observed in the right panel. At $L=20 \mathrm{~dB}$ SPL, the peak with a delay of $5.3 \mathrm{~ms}$ (hour-glass symbol) has the largest amplitude. As the stimulus level increases (at $L=30$ to $50 \mathrm{~dB}$ ), the peak with a delay of $4.2 \mathrm{~ms}$ (diamond symbol) has the largest amplitude, and at $L=60$ and $70 \mathrm{~dB}$ SPL, the peak with a delay of $1.3 \mathrm{~ms}$ (square symbol) has the largest amplitude.

An alternative analysis of the level series of the CR envelopes of Figure 10 is presented in Figure 11A. For each level series, responses to the lowest stimulus level were normalized to $0 \mathrm{~dB}$. Therefore, for each level series, stimulus level increases from the top to the bottom in the figure. The length of the lines indicates amplitude growth. The following features that were observed in Figure 10 are also evident in this figure: (1) persistence of delay peak across level, (2) decrease in amplitude peaks with increasing level, and (3) amplitude peaks with a wider dynamic range for the later occurring delay peaks.

Figure 11 also extends the level series analysis to eight other subjects. The qualitative analysis is consistent across subjects, although individual variability, more especially in the timings of the delay peaks, exists among subjects. The highly individual timings of the delay peaks and total number of peaks in a 


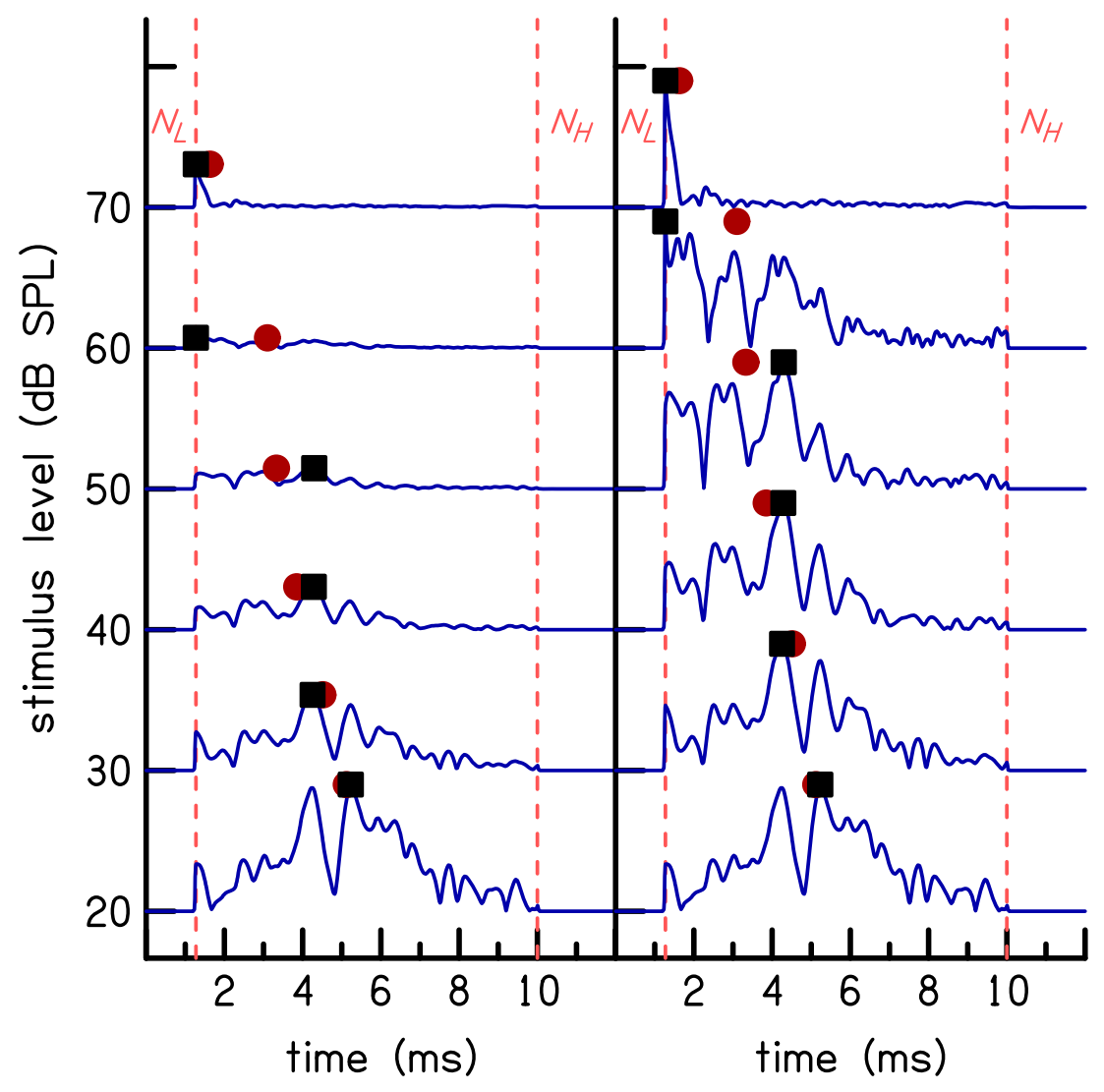

FIG. 9. Time-domain $C R$ envelopes at $4 \mathrm{kHz}$ and stimulus levels of $20-70 \mathrm{~dB}$ SPL for a single subject. The ordinate label shows the stimulus level and not the amplitude of the CR envelopes. CR envelopes on the right panel are normalized to the maximum peak for visual clarity. The dashed vertical lines are the limits $N_{L}$ and $N_{H}$. Superimposed on both panels are the envelope delay (filled squares) and group delay, $\tau_{\mathrm{CR}}$ (filled circles). Peaks persist across level, and the amplitudes of the peaks are largest at the lowest level. given time interval makes averaging results across subjects difficult. However, an analysis that averages the individual group delays is possible and is used to derive the latency-frequency relation.

\section{Latency-frequency relation}

The dependence of latency on frequency and stimulus level is evaluated by considering scatter plots of our estimates of group delay in the dimensionless quantity $N_{\mathrm{CR}}$ [cf. Eq. (8)], at different stimulus levels in Figure 12. Loess trend lines (Cleveland 1979) are also shown in the figure (solid lines) to guide the eye. A systematic variation in group delay with frequency can be visualized. Except for stimulus levels of 60 and $70 \mathrm{~dB}$ SPL, a breakpoint frequency, $f_{a \mid b}$ can be observed. Below this frequency, $N_{\mathrm{CR}}$ increases with increasing frequency and above, $N_{\mathrm{CR}}$ is near independent of frequency. The average value for the break point frequency is $f_{a \mid b}=1.5 \mathrm{kHz}$. This value was determined by finding the intersection of power-law fits to the steady-state low and high frequency portions of each latency-frequency curve. The breakpoint, or transition, frequency has been suggested to represent a transition between a high frequency region $\left(\beta>f_{a \mid b}\right)$ of more "basal-like" cochlea behavior and a lowfrequency region $\left(f<f_{a \mid b}\right)$ of more "apical-like" cochlea behavior (Shera et al. 2010).
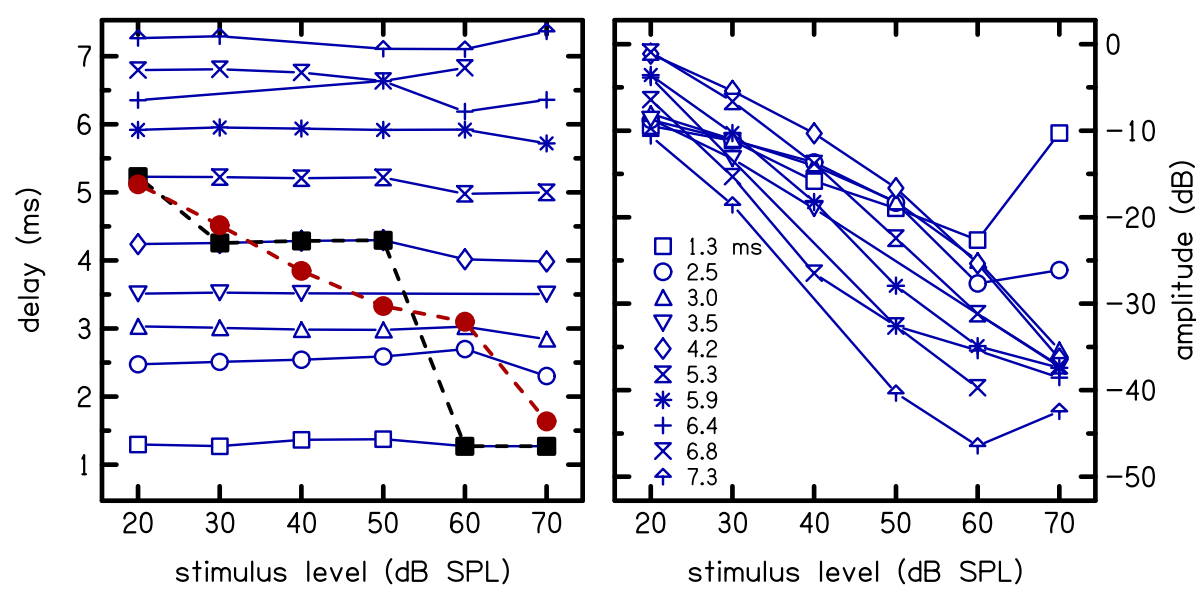

FIG. 10. Latency (left panel) and amplitude (right panel) of peaks shown in Fig. 9, as functions of stimulus level. Superimposed on the left panel are the envelope delay (filled squares) and group delay, $\tau_{C R}$ (filled circles). Only peaks with delays of up to the maximum of the envelope delay plus $2 \mathrm{~ms}$ are shown, to reduce clutter in the plot. Delay peaks persist across level. The amplitude of the peaks decrease with increasing stimulus level, with later occurring peaks having amplitudes with a wider dynamic range compared to earlier occurring peaks. 


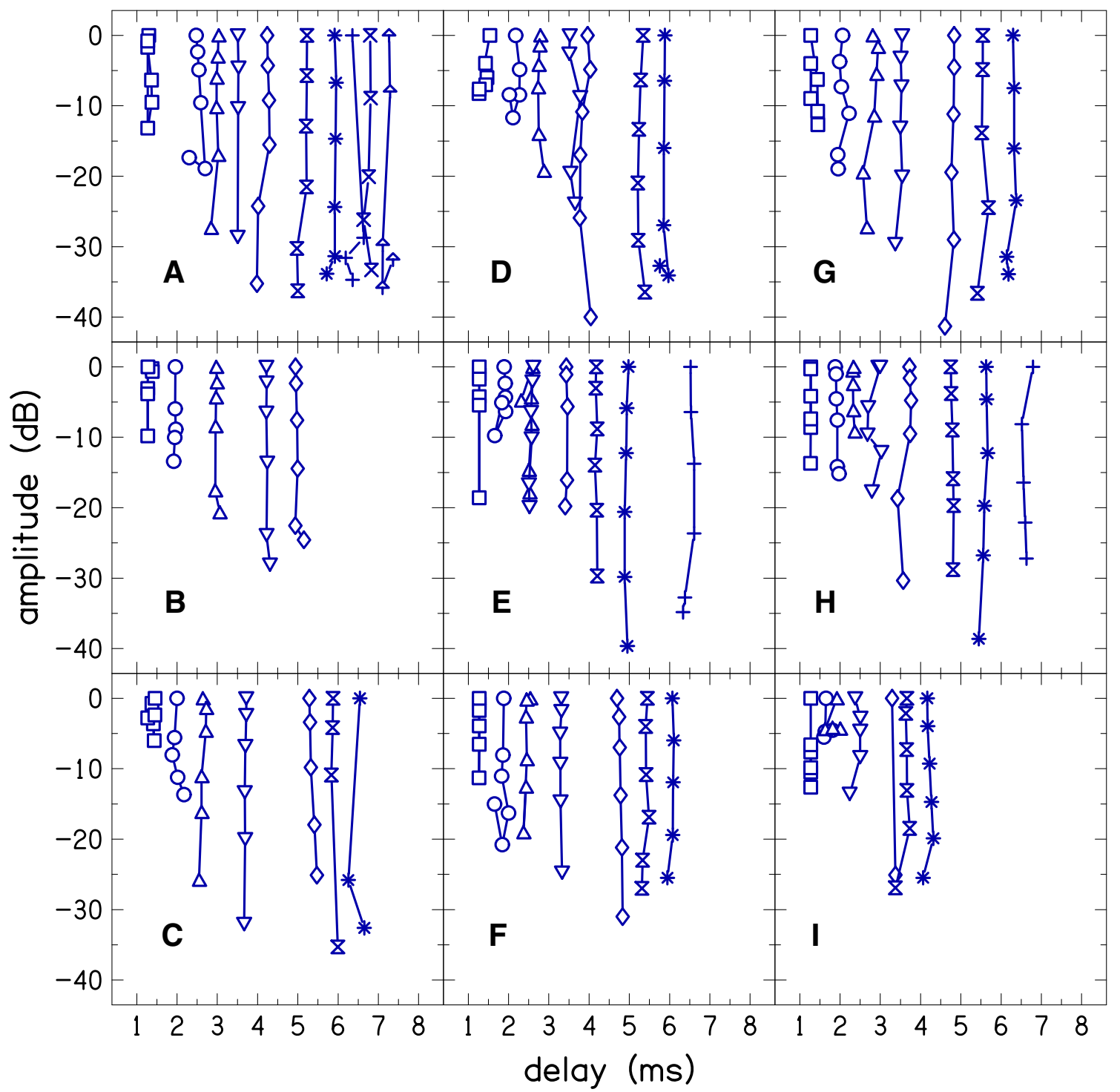

FIG. 11. Level series analysis for nine subjects. Stimulus level increases from top to bottom and the length of the lines indicates amplitude growth. The following observations can be generalized to the subjects (1) delay peaks persist across level, (2) amplitude of the peaks decrease with increasing stimulus level (movement from top to bottom), and (3) later occurring peaks have amplitudes with a wider dynamic range than the earlier occurring peaks.
The Loess trend lines for the latency-frequency functions $N_{\mathrm{CR}}$ in the different panels of Figure 12, except for $L=60$ and $70 \mathrm{~dB}$ SPL, are superimposed in Figure 13. The dependence of latency on stimulus level can be observed. For a given frequency, the latency is shorter at higher stimulus levels compared to lower levels. The slope of $N_{\mathrm{CR}}$ above $f_{a \mid b}$, but below $8 \mathrm{kHz}$, is near zero at all stimulus levels and the slope below $f_{a \mid b}$ ranges from 0.42 to 0.74 . There is an orderly decrease in slope below $f_{a \mid b}$ with increase in level. These slopes, determined via power law fits to the $N_{\mathrm{CR}}$ trend lines, are presented in Table 3. The latency functions of Figure 13 merge below $0.4 \mathrm{kHz}$ where there is uncertainty in the latency estimation due a reduction in CR energy at low frequencies (cf. Fig. 2).
The latency functions also converge at $16 \mathrm{kHz}$, which is probably influenced by the short time interval between the limits $N_{L}$ and $N_{H}$ at high frequencies. An equivalent plot of CR latency in millisecond versus frequency $\tau_{\mathrm{CR}}\left(f_{\mathrm{c}}\right)$ is shown in Figure 14 .

\section{DISCUSSION}

A method has been developed for obtaining CR from WBN-ECR measurements. CR constitutes mainly the nonlinear component of ECR and is equivalent to a measure of OAE normalized by the stimulus. The method includes two stages: (1) a subtraction procedure that removes middle-ear reflectance and (2) 


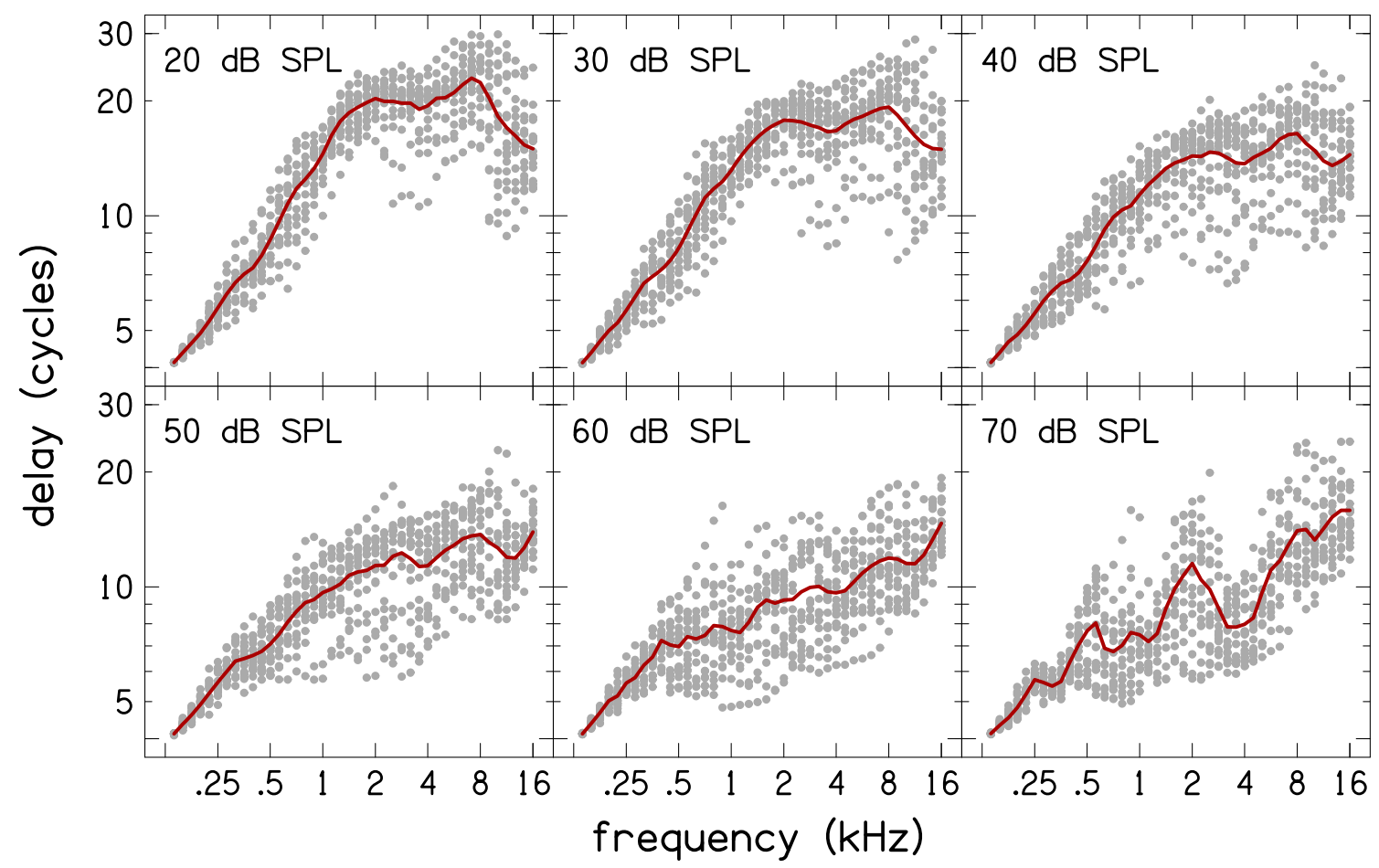

FIG. 12. $\quad N_{C R}$ versus frequency at different stimulus levels. The group delay $N_{C R}$ at each center frequency for all the subjects (dots) are plotted together for a given stimulus level, together with Loess trend lines to guide the eye. A breakpoint frequency $f_{\text {alb }}$ can be determined by inspection of the Loess trend lines.

time-frequency analysis to remove residual middleear reflectance and measurement-system artifact. Analyses were performed to examine the level dependence of latencies of individual peaks of time-domain $\mathrm{CR}$ and to examine the global dependence of CR on frequency and level. Use of noise to evoke OAEs has

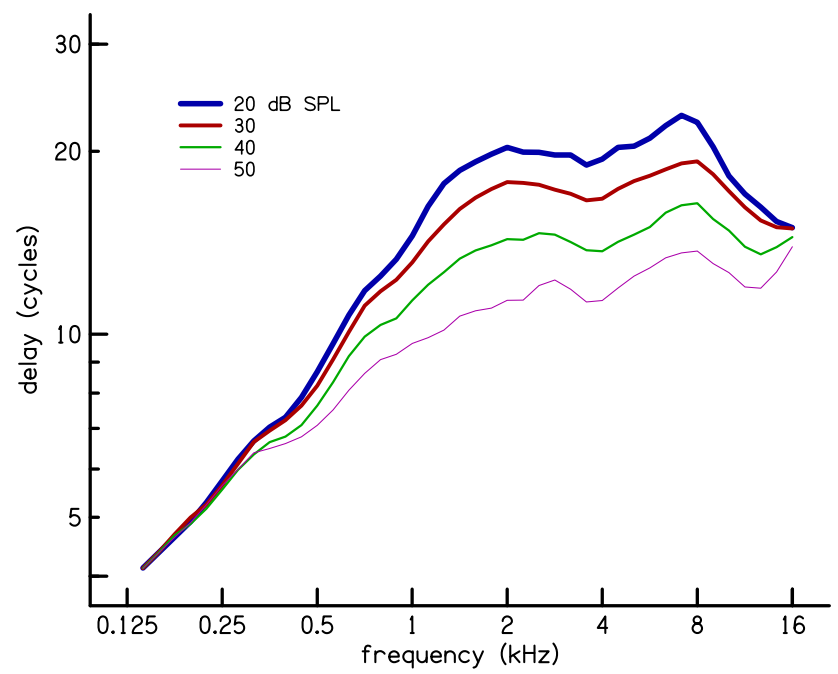

FIG. 13. Latency-frequency relation at stimulus levels of 20-50 dB $\mathrm{SPL}$. The Loess trend lines for the latency functions $N_{C R}$ at different stimuli levels are plotted together. Above the breakpoint frequency, $f_{a l b}$ $=1.5 \mathrm{kHz}, N_{\mathrm{CR}}$ has a near zero slope. The slopes of $N_{C R}$ below and above $f_{a l b}$ are presented in Table 3. CR delay is longer at low levels, compared to high levels. The latency estimates merge below $0.4 \mathrm{kHz}$, where there is uncertainty about the estimates. previously been done by Maat et al. (2000). However, the purpose of their study was to demonstrate that noise-evoked OAEs are similar to CEOAEs, and as such, their noise stimulus was band-limited to 0.8$5 \mathrm{kHz}$ (which is less than the bandwidth of our CR measurements) to match the spectrum of a click stimulus they used to evoke CEOAE. To the authors' knowledge, this is the only other study where noise was used to evoke OAEs.

In plots of time-domain CR obtaining using the subtraction procedure (cf. Fig. 1), high frequencycontent temporally precedes low-frequency content, which is consistent with signals of cochlea origin. These time-domain CR plots are similar to timedomain plots of CEOAE (e.g., Maat et al. 2000; Avan et al. 2000). Our CR though has a wider bandwidth,

TABLE 3

Slopes of the group delay function $N_{\mathrm{CR}}$ below and above the breakpoint frequency $f_{a l b}=1.5 \mathrm{kHz}$

\begin{tabular}{lcc}
\hline Stimulus level (dB SPL) & Slope below $f_{a l b}$ & Slope above $f_{a l b}$ \\
\hline 20 & 0.74 & 0.05 \\
30 & 0.65 & 0.02 \\
40 & 0.55 & 0.04 \\
50 & 0.42 & 0.09 \\
\hline
\end{tabular}

The slope of $N_{C R}$ was determine using data only up to $8 \mathrm{kHz}$. The slope of $N_{\text {CR }}$ above $f_{\text {alb }}$ is near zero. There seems to be an orderly decrease in slope below $f_{a l b}$ with increase in level. The slopes were determined via power law fits to the $N_{\mathrm{CR}}$ loess trend curves 


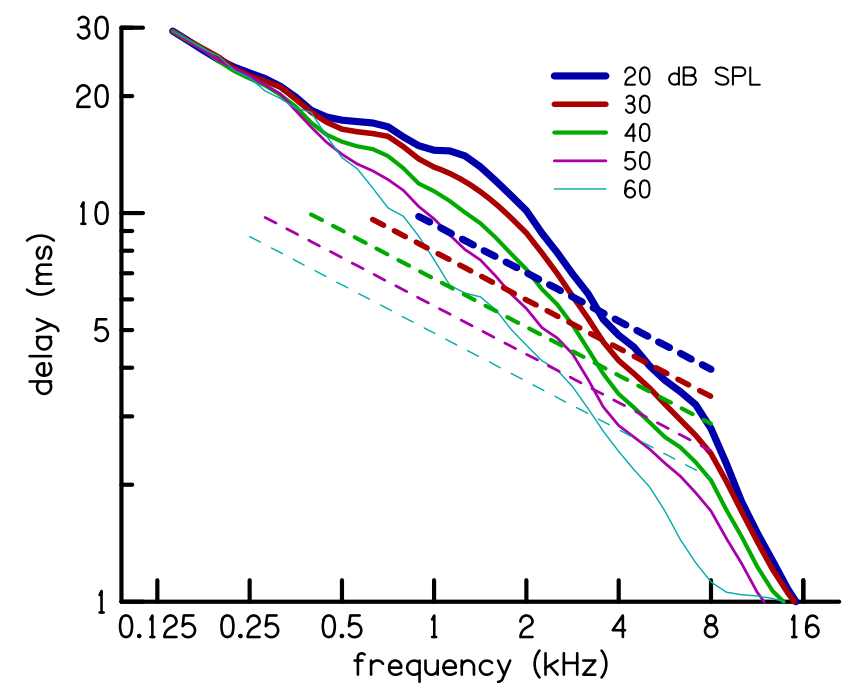

FIG. 14. Comparison of cochlear reflectance delay in milliseconds (solid lines) to tone-burst ABR forward latency (dashed lines) at stimulus levels of 20-60 dB SPL. The decrease in CR latency per decibel increase in level is similar to that of ABR forward latency between 2 and $8 \mathrm{kHz}$.

when compared to CEOAEs measured in humans using the commonly used nonlinear residual method, which discards the short latency part of the response (e.g., Kemp et al. 1986). However, the double-evoked CEOAE paradigm described by Keefe et al. (2011) has a similarly wide bandwidth of up to $16 \mathrm{kHz}$. The wide bandwidth of our time-domain CR allows for measurement of CR magnitude and latency that represent more than $80 \%$ of the length of the cochlea (Greenwood 1990).

Gammatone filterbanks were used in the timefrequency analysis to separate CR from residual middle-ear reflectance and measurement-system artifact. A gammatone filterbank has not been used for OAE analysis in previous studies. Other time-frequency analysis techniques, such as the short-time Fourier transform and wavelet transform, may also be used instead of the gammatone filterbank and will produce similar results. The gammatone filterbank, like most other filters, introduces dispersion to the reflectance; however, this effect is small.

The limits $N_{L}$ and $N_{H}$ [cf. Eqs. (3) and (4)] are important for separating middle-ear activity from cochlea activity, and their settings were carefully selected based on inspection of a large number of gammatone spectrograms. These limits do not bias the estimation of latency significantly because an inspection of a large number of gammatone spectrograms showed that most of the CR is included within these limits. The residual activity in the CR before application of time-frequency is probably due to variations in time caused by shifts in the position of the measurement probe between measurements. The limit $N_{L}$ provides a good separation between cochlear contributions and middle-ear and measurement-system activity, except maybe at high frequencies (above $8 \mathrm{kHz}$ ), where there are possible short delay contributions from the cochlear base. This ambiguity in the separation of cochlear reflectance and residual activity may have been responsible for the convergence observed in the CR latency estimates at $16 \mathrm{kHz}$.

The notches in the CRM of Figure 6 are not as sharp as notches that have been observed in magnitude spectra of OAEs and transfer functions of OAEs (e.g., Kalluri and Shera 2007; Norton and Neely 1987; Sisto and Moleti 2008) because our reflectance magnitude computation method [cf. Eq. (10)] smoothes out the sharpness of the notches, as demonstrated in Figure 3. However, the smoothed spectrum still reveals the dependence of CRM on stimulus level and frequency and facilitates comparison of spectra across different stimulus levels. The sharpness of the notches of CRM can be increased either by decreasing the bandwidth of the filters in the filterbank analysis or using a time-frequency analysis method that offers perfect reconstruction, such as wavelets and the short-time Fourier transform. Mean CRM as a function of frequency (cf. Fig. 7) has a peak at $1.1 \mathrm{kHz}$ that has been observed in previous OAE measurements (e.g., Sisto and Moleti 2008). This peak is probably the effect of round-trip transmission through the middle ear as was previously demonstrated by Puria (2003) and Sisto and Moleti (2008).

CRM as a function of stimulus level has a constant slope of $-0.6 \mathrm{~dB} / \mathrm{dB}$ for intermediate levels and saturates at high levels (cf. Fig. 8). Gorga et al. (2011) observed a slope of about $-0.6 \mathrm{~dB} / \mathrm{dB}$ in their estimation of cochlear-amplifier gain using tip-to-tail differences of DPOAE suppression tuning curves. This similarity suggests that CRM decreases with increase in level because the cochlear-amplifier gain decreases. CRM saturates at the higher stimulus levels and becomes independent of stimulus level because at these levels the cochlear-amplifier is not as active (e.g., Kemp 2002; Harte et al. 2009). The acoustic reflex, which causes impedance mismatch in the middle ear and decreases the transmission of energy to the cochlea, may also be responsible for the reduction in CRM at the higher levels. At low levels, the slope of the CRM becomes shallower $(-0.48 \mathrm{~dB} / \mathrm{dB})$, suggesting onset of cochlear linearity. Additional measurements at levels below $20 \mathrm{~dB}$ SPL are required to determine the low-level linear range of CR.

The level-series analysis of time-domain components of CR (cf. Figs. 9, 10 and 11) revealed that (1) delay peaks persist across level with timings unchanged, (2) amplitude of the peaks decrease with increasing stimulus level, and (3) later occurring peaks have amplitudes with a wider dynamic range than earlier occurring peaks. The persistence of the 
timings of the peaks can also be noted in Figure 1. Our last two observations may seem to contradict those of Stover et al. (1996) and Goodman et al. (2011). In their temporal analysis of DPOAE, Stover et al. (1996) noted that the amplitudes of early occurring peaks had a wider dynamic range than those of later occurring peaks and that amplitude of peaks grow with stimulus level. Goodman et al. (2011) made similar observations in their time-domain analysis of TEOAE. However, CR is a form of an otoacoustic emission that is normalized by the stimulus. When this is taken into consideration, our observations are consistent with these earlier observations. We believe that the multiple delay peaks do not signify multiple sources of OAE generation along the BM, but are due to interference and re-reflections of delayed signals coming back from various points along the BM.

In the level-series analysis and in general, the group delay is different from the envelope delay because the group delay represents a weighted average delay of all the peaks, not just the dominant peak. However, at the lower stimulus levels where the amplitude of the dominant peak is much greater than the amplitude of the other peaks, the group delay and envelope delay are similar. The similarities of the results of our level series analysis to previous results indicate that our measurements are indeed of cochlear origin.

Our measurements of CR can be considered as a superposition of multiple SFOAE measurements that have been normalized by the stimulus. In terms of the two OAE generator mechanisms, CR can then be considered to be due to linear coherent reflection. However, an important difference between the CR and SFOAE exists. SFOAE is typically measured using a pure tone at a single frequency, which, at low stimulus levels, excites a spatially limited region of the cochlea. On the other hand, the wideband stimulus used to measure CR simultaneously excites an extensive region of the cochlea, perhaps the entire cochlea, at the same time. This brings up possibilities for intermodulation products generated by interaction between different frequency components of the stimulus, as was observed by Yates and Withnell (1999) for TEOAEs. Although this complicates the comparison of our measurements to current measures of OAE, the advantage of using a wideband noise stimulus is that it allows for the invocation of the EQNL theorem (de Boer 1997), which simplifies the interpretation of CR. The EQ-NL theorem allows the nonlinear mechanics of the cochlea to be replaced by an equivalent linear system for each stimulus level. Linear systems are easier to analyze than nonlinear systems because most analysis tools (e.g., transfer function, Fourier analysis) assume linearity.

The dependence of CR latency on frequency and level is consistent with earlier estimates of OAE and auditory brainstem response (ABR) latency (e.g., Neely et al. 1988; Harte et al. 2009). A comparison of CR latency to tone-burst ABR forward latency of Neely and Gorga (1998) is shown in Figure 14. The decrease in ABR forward latency per decibel increase in level is $1.62 \%$, at all levels and frequencies. In the current measurements, CR latency between 2 and $8 \mathrm{kHz}$ and for level below $50 \mathrm{~dB}$ SPL decreases by a mean value of $1.65 \%$ per $\mathrm{dB}$ increase in level, giving further support to our measurements being of cochlear origin. However, the fact that CR latency is not proportional to twice the ABR forward latency (except at approximately $1 \mathrm{kHz}$ ) brings into question the prevailing interpretation of OAE latency as being twice the travel time of a particular frequency component from stimulus onset through the cochlea to its characteristic place. Another distinct feature of ABR latencies is that they do not have any apparent "breakpoint frequency" as has been observed for CR and other OAE latencies. We do not yet have clear answers to these questions.

The observed breakpoint in the latency (cf. Fig. 12) is also consistent with earlier studies of OAEs in humans and other mammals (Shera and Guinan 2003; Shera et al. 2010; Schairer et al. 2006; Bergevin et al. 2008; Dhar et al. 2011). Our breakpoint frequency of $1.5 \mathrm{kHz}$ agrees exactly with recent estimates by Dhar et al. (2011) and Abdala and Dhar (2012) using DPOAE. Although there are difference in the breakpoint frequencies compared by the other investigators, the breakpoint frequencies all occurs near the mid-point of the cochlea (Shera et al. 2010). In the current study, the observed breakpoint of $1.5 \mathrm{kHz}$ maps to a distance of $1.8 \mathrm{~cm}$ from the stapes according to the Greenwood frequency-place map (Greenwood 1990), which is the midpoint since a typical length of a human cochlea is about $3.5 \mathrm{~cm}$. The breakpoint, or transition, frequency has been suggested to represent a transition between a highfrequency region $\left(\beta f_{a \mid b}\right)$ of more "basal-like" cochlea behavior and a low-frequency region $\left(f<f_{a \mid b}\right)$ of more "apical-like" cochlea behavior (Shera et al. 2010). For frequencies above the breakpoint $f_{a \mid b}$, the slope of the latency-frequency function $N_{\mathrm{CR}}$ is near zero, suggesting that the cochlea has scaling symmetry (Shera et al. 2000). However, scaling symmetry may still hold below the breakpoint frequency, despite a non-zero slope in the delay if the reflection occurred at a basal tail of a cochlear travelling wave with a scaling-symmetric excitation pattern (Choi et al. 2008).

The slopes of the latency-frequency function below the breakpoint frequency decreases with increasing stimulus level (cf. Fig. 13 and Table 3). This decline in latency slope with increasing level was also noted by Sisto and Moleti (2007) for TEOAEs. However, it is possible that the merging of the latency functions 
below $0.4 \mathrm{kHz}$ may have influenced the observed decline in latency slope, and perhaps, the slopes at the different levels are actually similar.

\section{CONCLUSION}

CR measured in this study has features that are characteristic of signals of cochlear origin. The CR measurements offer three advantages over other types of otoacoustic emissions: (1) wider bandwidth, (2) minimal influence from the measurement system and middle-ear on the recorded emission, and (3) easier interpretation due to the existence of an equivalent linear model, which validates the application of linearsystems theory. CR provides an innovative and potentially informative means for assessing cochlear processing, compared to other noninvasive examinations of the human cochlea.

\section{ACKNOWLEDGMENTS}

This research was supported by grants from NIH (grants nos. R01 DC8318 and P30 DC4662). We would like to thank Cori Birkholz, Alyson Gruhlke, and Judy Kopun for their help in recruiting subjects and making measurements. We would like to thank Jonathan $\mathrm{H}$. Siegel for providing us with the sound-delivery equipment. Finally, we thank Joyce Rodriquez, Michael P. Gorga, Christopher A. Shera, and three anonymous reviewers for helpful suggestions on earlier versions of this manuscript.

\section{Open Access}

This article is distributed under the terms of the Creative Commons Attribution License which permits any use, distribution, and reproduction in any medium, provided the original author(s) and the source are credited.

\section{REFERENCES}

Abdala C, Dhar S (2012) Maturation and aging of the human cochlea: a view through the DPOAE looking glass. J Assoc Res Otolaryngol 13:403-421

Allen JB (1986) Measurement of eardrum acoustic impedance. In: Allen JB, Hall Jl, Hubbard A, Neely ST, Tubis A (eds) Peripheral auditory mechanisms. Springer, New York

Allen JB (1997) Derecruitment by multiband compression in hearing aids. In: JESTEAdT W (ed) Modeling sensorineural hearing loss. Lawrence Erlbaum Associates, Mahwah, pp 99-112

American National Standards Institute (1996) Specifications for audiometers. ANSI S3.6-1996, New York

Avan P, Wit HP, Guitton H, Mom T, Bonfils P (2000) On the spectral periodicity of transient-evoked otoacoustic emissions from normal and damaged cochleas. J Acoust Soc Am 108:11171127
Bergevin C, Freeman DM, Saunders JC, Shera CA (2008) Otoacoustic emissions in humans, birds, lizards, and frogs: evidence for multiple generation mechanisms. J Comp Physiol A 194:665-683

Choi YS, Lee SY, Parham K, Neely ST, Kim DO (2008) Stimulusfrequency otoacoustic emission: measurements in humans and simulations with an active cochlear model. J Acoust Soc Am 123:2651-2669

Claerbout J (1985) Imaging the Earth's interior. Blackwell Scientific, Palo Alto, pp 287-289

Cleveland WS (1979) Robust locally weighted regression and smoothing scatterplots. J Am Stat Assoc 74:829-836

DE BOER E (1997) Connecting frequency selectivity and nonlinearity for models of the cochlea. Audit Neurosci 3:377-388

Dhar S, Rogers A, Abdala C (2011) Breaking away: violation of distortion emission phase-frequency invariance at low frequencies. J Acoust Soc Am 129:3115-3122

Goldstein JL, BaER T, Kiang NYS (1971) A theoretical treatment of latency, group delay, and tuning characteristics of auditory-nerve responses to clicks and tones. In: SACHS MB (ed) Physiology of the auditory system. National Educational Consultants, Baltimore, pp 133-141

Goodman SS, Mertes IB, Scheperle RA (2011) Delay and growth rate of multiple TEOAE components. In: SHERA CA, OLSON E (ed) What fire is in mine ears: progress in auditory biomechanics. Am Inst Phys, New York, pp 279-284

Gorga MP, Neely ST, Kopun J, Tan H (2011) Distortion-product otoacoustic emission suppression tuning curves in humans. J Acoust Soc Am 129:817-827

GREENwOOD DD (1990) A cochlear frequency-position function for several species-29 years later. J Acoust Soc Am 87:2592-2605

Нӓrмä A (1999) Derivation of the complex-valued gammatone filters. http://www.acoustics.hut.fi/software/HUTear/Gammatone/ Complex_gt.html. Accessed 2 Apr 2012

Harte JM, Pigasse G, Dau T (2009) Comparison of cochlear delay estimates using otoacoustic emissions and auditory brainstem responses. J Acoust Soc Am 126:1291-1301

Hohmann V (2002) Frequency analysis and synthesis using a gammatone filterbank. Acustica 88:433-442

Jepsen ML, Ewert SD, Dau T (2008) A computational model of human auditory signal processing and perception. J Acoust Soc Am 124:422-438

Kalluri R, Shera CA (2007) Near equivalence of human clickevoked and stimulus-frequency otoacoustic emissions. J Acoust Soc Am 121:2097-2110

KeEFe DH, Schairer KS (2011) Specification of absorbed-sound power in the ear canal: application to suppression of stimulus frequency otoacoustic emissions. J Acoust Soc Am 129:779-791

Keefe DH, Ling R, Bulen RC (1992) Method to measure acoustic impedance and reflection coefficient. J Acoust Soc Am 91:470-485

Keefe DH, Goodman SS, Ellison JC, Fitzpatrick DF, Gorga MP (2011) Detecting high-frequency hearing loss with click-evoked otoacoustic emissions. J Acoust Soc Am 129:245-261

Kemp DT (2002) Exploring cochlear status with otoacoustic emissions, the potential for new applications. In: RoBINETTE MS, Glattke TJ (eds) Otoacoustic emissions: clinical applications, 2nd edn. Thieme Medical, New York, pp 1-47

Kemp DT, Bray P, Alexander L, Brown AM (1986) Acoustic emission cochleography-practical aspects. Scand Audiol Suppl 25:71-95

MaAt B, Wit HP, van Dijk P (2000) Noise-evoked otoacoustic emissions in humans. J Acoust Soc Am 108:2272-2280

Meddis R, O'Mard LP, Lopez-Poveda EA (2001) A computational algorithm for computing nonlinear auditory frequency selectivity. J Acoust Soc Am 109:2852-2861

Neely ST, Gorga MP (1998) Comparison between intensity and pressure as measures of sound level in the ear canal. J Acoust Soc Am 104:2925-2934 
Neely ST, Liu Z (1994) EMAV: otoacoustic emission average. Technical Memo No. 17. Boys Town National Research Hospital, Omaha

Neely ST, Norton S, Gorga MP, Jesteadt W (1988) Latency of auditory brain-stem responses and otoacoustic emissions using tone-burst stimuli. J Acoust Soc Am 83:652-656

Norton SJ, Neely ST (1987) Tone-burst-evoked otoacoustic emissions from normal-hearing subjects. J Acoust Soc Am 81:1860-1872

Patterson RD, Holdsworth J (1996) A functional model of neural activity patterns and auditory images. In: AINSWORTH AW (ed) Advances in speech, hearing and language processing. JAI, London, pp 547-563

PURIA S (2003) Measurements of human middle ear forward and reverse acoustics: Implications for otoacoustic emissions. J Acoust Soc Am 113:2773-2789

Rasetshwane DM, Neely ST (2011) Inverse solution of ear-canal area function from reflectance. J Acoust Soc Am 130:38733881

Rhode WS, Robles L (1974) Evidence from Mössbauer experiments for nonlinear vibration in the cochlea. J Acoust Soc Am 55:588596

Rosowski JJ, Nakajima HH, Hamade MA, Mahfoud L, Merchant GR, Halpin CF, Merchant SN (2012) Ear-canal reflectance, umbo velocity, and tympanometry in normal-hearing adults. Ear Hear 33:19-34

Schairer KS, Ellison JC, Fitzpatrick D, Keefe DH (2006) Use of stimulus-frequency otoacoustic emission latency and level to investigate cochlear mechanics in humans. J Acoust Soc Am 120:901-914

Scheperle RA, Goodman SS, NeEly ST (2011) Further assessment of forward pressure level for in situ calibration. J Acoust Soc Am 130:3882-3892

Shera CA, Guinan JJ (1999) Evoked otoacoustic emissions arise by two fundamentally different mechanisms: a taxonomy for mammalian OAEs. J Acoust Soc Am 105:782-798
Shera CA, Guinan JJ (2003) Stimulus-frequency-emission group delay: a test of coherent reflection filtering and a window on cochlear tuning. J Acoust Soc Am 113:2762-2772

Shera CA, Talmadge CL, Tubis A (2000) Interrelations among distortion-product phase-gradient delays: their connection to scaling symmetry and its breaking. J Acoust Soc Am 108:2933-2948

Shera CA, Tubis A, Talmadge CL (2005) Coherent reflection in a two-dimensional cochlea: short-wave versus long-wave scattering in the generation of reflection-source otoacoustic emissions. J Acoust Soc Am 118:287-313

Shera CA, Tubis A, Talmadge CL (2008) Testing coherent reflection in chinchilla: auditory-nerve responses predict stimulus-frequency emissions. J Acoust Soc Am 124:381-395

Shera CA, Guinan JJ, Oxenham AJ (2010) Otoacoustic estimation of cochlear tuning: validation in the chinchilla. J Assoc Res Otolaryngol 11:343-365

Siegel JH (1994) Ear-canal standing waves and high-frequency sound calibration using otoacoustic emission probes. J Acoust Soc Am 95:2589-2597

Siegel JH, Cerka AJ, Recio-Spinoso A, Temchin AN, van Dijk P, RUGGero MA (2005) Delays of stimulus-frequency otoacoustic emissions and cochlear vibrations contradict the theory of coherent reflection filtering. J Acoust Soc Am 118:2434-2443

Sisto R, Moletr A (2007) Transient evoked otoacoustic emission latency and cochlear tuning at different stimulus levels. J Acoust Soc Am 122:2183-2190

Sisto R, Moleti A (2008) Transient evoked otoacoustic emission input/output function and the cochlear reflectivity: experiment and model. J Acoust Soc Am 124:2995-3008

Stover LJ, Neely ST, Gorga MP (1996) Latency of multiple sources of distortion product otoacoustic emissions. J Acoust Soc Am 99:10161024

Voss SE, Allen JB (1994) Measurement of acoustic impedance and reflectance in the human ear canal. J Acoust Soc Am 95:372-384

Yates GK, WithNelL RH (1999) The role of intermodulation distortion in transient-evoked otoacoustic emissions. Hear Res 136:49-64 